\title{
Fuzzy4U : un moteur d'adaptation en logique floue pour l'accessibilité des interfaces utilisateurs
}

\section{Tanguy Giuffrida \\ Université Grenoble Alpes, CNRS, Grenoble INP*, LIG 38000 Grenoble, France. \\ tanguy.giuffrida@univ-grenoble- alpes.fr \\ Éric Céret \\ Université Grenoble Alpes, CNRS, Grenoble INP*, LIG 38000 Grenoble, France. \\ Sophie Dupuy-Chessa \\ Université Grenoble Alpes, CNRS, Grenoble INP*, LIG 38000 Grenoble, France. \\ Jean-Philippe Poli \\ CEA LIST \\ Laboratoire Analyse de Données et Intelligence Artificielle 91191 Gif-sur-Yvette, France. \\ * Institute of Engineering Univ. Grenoble Alpes}

\section{Fuzzy4U: A fuzzy logic adaptation engine for interfaces' accessibility}

Abstract. With the massive spread of Internet use, the accessibility of user interfaces (UI) is an ever more pressing need. Much work has been developed on this subject in order to define generic or situational accessibility recommendations and to propose tools for user interface adaptation. However, difficulties remain, particularly related to the complexity of possible contexts of use, such as the multiplicity of characteristics of the context of use, the imprecision of the values assigned to these characteristics and the combination of multiple adaptation rules. This article shows how a dynamic adaptation engine based on fuzzy logic can be used to implement accessibility recommendations. We show how this approach makes it possible to overcome these difficulties through fuzzy logic with the capacity to manage combinatorial rules, making it possible to take into account potentially complex contexts of use. This approach is illustrated with a concrete example.

Key words: User Interface adaptation, fuzzy logic, accessibility, context of use, adaptation rules

Résumé. Avec la diffusion massive de l'utilisation d'Internet, l'accessibilité des interfaces est un besoin toujours plus prégnant. De nombreux travaux se sont penchés sur ce sujet afin de définir des recommandations d'accessibilité génériques ou situationnelles, et proposer des outils d'adaptation des interfaces utilisateurs. Cependant, des difficultés, notamment liées à la complexité des contextes d'usage possibles, demeurent tels que la multiplicité des caractéristiques du contexte d'usage, l'imprécision des valeurs attribuées à ces caractéristiques et la combinaison de multiples règles d'adaptation. Cet article montre comment un moteur d'adaptation dynamique basé sur la logique floue peut être utilisé pour implémenter les préconisations en accessibilité. II montre comment cette approche permet de dépasser ces verrous grâce à la logique floue et sa gestion de la combinatoire des règles, permettant de prendre en compte un contexte d'usage potentiellement complexe que nous illustrons avec un exemple concret.

Mots-clés: Adaptation d'Interface Utilisateur, logique floue, accessibilité, contexte d'usage, règles d'adaptation 


\section{INTRODUCTION}

L'utilisation de l'informatique s'impose de plus en plus dans la vie quotidienne, notamment à travers Internet. On peut penser, par exemple, à la diffusion massive des réseaux sociaux, à l'accès aux comptes bancaires, aux déclarations d'impôts, aux prises de rendez-vous médicaux.

En France, le code de l'action sociale et des familles promeut l'utilisation des technologies numériques pour prévenir l'isolement, notamment dans l'article L233-1 de la loi 2015-1776 du 28 décembre 2015. Pourtant, le Haut Conseil de la famille, de l'enfance et de l'âge, dans son rapport du 16 mai 2019 (HCFEA, 2019), note l'existence d'une "fracture numérique" qui atteint "certains territoires [et] certains publics fragiles". Cette fracture retranscrit la difficulté d'une part importante de la population à utiliser l'outil informatique et constitue un facteur supplémentaire d'isolement. Ces personnes - notamment les personnes en difficulté neurocognitive ou les personnes âgées - montrent en effet une forte anxiété et un sentiment d'incompétence vis-à-vis de ces dispositifs (Quillion-Dupré et al., 2018). Par rapport à la population générale, elles ont besoin de plus de temps pour s'approprier l'utilisation d'une nouvelle technologie et requièrent plus d'aide et d'entraînement (QuillionDupré et al., 2018).

Si l'accompagnement humain est un facteur important de succès pour permettre à ces personnes d'accéder à l'usage des outils informatiques (Berner, 2014), la facilité d'utilisation des dispositifs est un point critique pour en favoriser l'adoption (Alm et al., 2007) : les difficultés vécues par ces utilisateurs posent de façon plus aiguë la question de l'accessibilité. L'accessibilité est définie par le W3C comme les moyens à mettre en œuvre pour «permettre un accès égal et des opportunités égales à tous les utilisateurs ", afin notamment de « rendre possible une égale participation des personnes handicapées » (WAI, 2005).

Cependant, la Classification Internationale pour le Fonctionnement, le Handicap et la Santé de l'OMS insiste fortement sur le fait que le handicap est une limite imposée à l'engagement de la personne dans des activités, révélée, accrue ou allégée par des facteurs socio-environnementaux persistants ou ponctuels. Ainsi une personne atteinte de dégradation neurocognitive pourrait confortablement préparer ses repas ou discuter avec un membre de sa famille (des routines acquises depuis des années) tandis qu'elle aurait le plus grand mal à acquérir les bases d'usage d'un dispositif informatique: il s'agit alors d'une situation persistante. Une autre personne pourrait avoir assimilé l'envoi de mèls, mais se trouver totalement impuissante lorsque le système lui afficherait un message urgent concernant une mise à jour, une difficulté ponctuelle ou un handicap situationnel (Wobbrock, 2019).

Au regard de ces situations, le besoin d'accompagnement est double : les dispositifs informatiques doivent prendre en compte non seulement les préférences de l'utilisateur, mais aussi ses limitations, qu'elles soient durables ou ponctuelles. En d'autres termes, l'adaptativité dynamique et situationnelle des interfaces utilisateurs devient un élément crucial des systèmes du futur.

Pourtant, les préconisations, comme nous le détaillerons dans la section 5 , sont souvent des principes généraux ("le contenu doit être lisible par un enfant" d'environ 14 ans (Loranger, 2017)) et statiques ("le ratio de contraste recommandé est 4,5:1" (WCAG, 2009)), parfois complexes à combiner, voire contradictoires. Adapter une interface utilisateur à la situation, au handicap ponctuel, ne peut pas se faire sur la base de principes statiques: l'interface utilisateur doit réagir à la situation présente avec toute sa complexité, pour maintenir le niveau de service offert et préserver l'utilisabilité (Thevenin et Coutaz, 1999).

De nombreux travaux ont déjà traité des Interfaces Homme-Machine (IHM) adaptatives, des années 80 (Edmonds, 1981 ; Schneider-Hufschmidt et al., 1993 ; Kolski et al., 1998), jusqu'à récemment (Sottet et al., 2007 ; Blumendorf et al., 2008 ; Chesta et al., 2004 ; 
Florins, 2006 ; Nguyen et al., 2016). Néanmoins, ces approches sont souvent complexes, comme l'illustrent les travaux en Ingénierie Dirigée par les Modèles (IDM) (Whittle et al., 2014), largement explorées dans la littérature (Thevenin et al., 2003 ; Miñon et al., 2016 ; Paternò et al., 2009). De plus, des verrous scientifiques demeurent, en particulier si l'adaptation prend en compte l'utilisateur et pas seulement la plate-forme et l'environnement (Miñón et al., 2016 ; Yigitbas et al., 2017 ; Abrahâo et al., 2017). Nous avons établi qu'il reste, a minima, trois verrous :

(a) la multiplicité des caractéristiques du contexte d'usage (le triplet <utilisateur, plateforme, environnement>, (Calvary et al., 2003)), et en particulier du modèle de l'utilisateur : le cluster Virtual User Modelling and Simulation (VUMS, 2013) a identifié plus de 250 caractéristiques utiles pour simuler un utilisateur humain, en ne focalisant que sur les caractéristiques physiques de l'humain ;

(b) l'imprécision des valeurs attribuées à ces caractéristiques, que cette évaluation soit faite par capture (on peut par exemple inférer l'âge de l'utilisateur à partir d'une image prise par sa caméra, mais la précision est inférieure à 50\% (Jung et al., 2018)) ou par déclaration (on peut demander à l'utilisateur de déclarer son acuité visuelle, mais l'incertitude sur la valeur collectée est ici aussi élevée (Jasberg et al., 2018)) ;

(c) la combinaison des règles d'adaptation: prendre en compte les multiples caractéristiques du contexte d'usage nécessite, sous une forme ou une autre (transformations de modèles, patrons de solutions, règles logiques, etc.), de définir de multiples règles, puis de les combiner pour obtenir une solution en résolvant à la fois le compromis entre les différentes règles et la gestion performante de cette combinatoire (Yang et Shao, 2007).

Avec l'objectif d'aborder ces trois verrous, nous proposons une approche basée sur la logique floue pour gérer l'adaptation au contexte d'usage. Notre approche a fait l'objet d'une première évaluation par des experts (Giuffrida et al., 2019). Nous focalisons ici sur l'intérêt de notre approche pour l'accessibilité.

Nous commençons par présenter la notion d'accessibilité, puis l'état de l'art en section 3 , avant d'introduire en section 4 les concepts de la logique floue. La section 5 présente l'architecture logicielle de notre solution. Nous présentons ensuite, en section 6 , notre prise en compte des préconisations d'accessibilité suivie, en section 7, par leur mise en œuvre, avant de conclure par la discussion de la section 8.

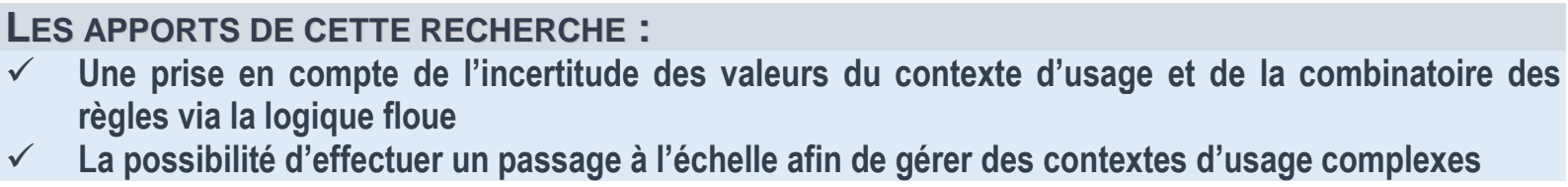

\section{ACCESSIBILITE}

\subsection{La notion d'accessibilité}

La notion d'accessibilité fait l'objet d'un travail important et ancien du World Wide Web Consortium (W3C), qui la décline dans plusieurs documents. Nous-nous sommes principalement appuyés 1) sur les règles normatives du W3C, présentées dans le Web Content Accessibility Guidelines (WCAG) de 2009 qui contient des recommandations génériques sur l'accessibilité ; 2) sur les Exigences d'accessibilité pour les personnes malvoyantes (abrégé EAPM dans la suite du document) de 2016 qui décrivent les besoins des personnes malvoyantes pour que le contenu, les outils et les technologies électroniques leur soient accessibles; et 3) sur les recommandations du projet européen Web Accessibility Initiative: Ageing Education and Harmonisation (WAI-AGE) de 2010 qui permettent d'augmenter l'accessibilité du web pour les personnes âgées ainsi que pour les personnes 
en situation de handicap. Ces deux derniers documents sont des documents de travail du W3C.

Dans cet article, nous présentons les préconisations concernant l'accessibilité, en particulier l'accessibilité du Web, qui a fait l'objet d'un plus grand nombre d'études en raison de la généralisation de son usage. L'accessibilité du web peut en effet être vue comme une problématique d'adaptation au contexte: certaines personnes ou certaines situations entraînent des besoins particuliers que l'adaptation peut aider à traiter.

\subsection{Exemple fil rouge}

Bernard a 79 ans. De façon très classique à cet âge, sa vision est dégradée et il est atteint de cataracte. Cette affection induit une nette diminution de sa perception des couleurs (daltonisme) et une légère photophobie (sensibilité douloureuse aux couleurs trop lumineuses). Bernard a donc l'habitude de grossir les polices de caractères lorsqu'il consulte un site Internet. II souffre aussi de douleurs articulaires qui l'obligent à utiliser une canne pour marcher. Mais Bernard reste actif et vient de partir en voyage à Lyon. II souhaite résider dans un hôtel qu'on lui a recommandé, et doit utiliser le métro pour se rendre de la Gare de Part-Dieu à cet hôtel. II ne connaît pas très bien la ville, mais sa fille lui a montré que l'hôtel propose une page décrivant les moyens d'accès. Bernard souhaite donc consulter cette page à la fois en entrant dans le métro, pour être sûr de la ligne et de l'arrêt, et à la sortie du métro pour se diriger jusqu'à l'hôtel.

Cet exemple illustre les règles suivantes d'adaptation dans le but de garantir l'accessibilité :

- En raison d'une acuité visuelle amoindrie : la taille de la police de caractères est augmentée, les espacements entre lettres, entres mots et entre lignes sont augmentés ;

- Si l'utilisateur est un senior : la taille de police de caractères est augmentée, la taille de police de caractères des liens est augmentée, la luminosité du fond d'écran est éclaircie, les espacements entre mots, entres lignes et entre paragraphes sont augmentés ;

- Si l'utilisateur est daltonien ou s'il a une autre forme de diminution de perception des couleurs : les couleurs sont modifiées afin de remplacer celles qui sont mal perçues ;

- En cas de photophobie, la luminosité du fond d'écran est assombrie, le niveau de contraste est réduit ;

- Si la luminosité ambiante est forte (plein soleil) : la luminosité du fond d'écran est éclaircie, le niveau de contraste est augmenté ;

- Si la luminosité ambiante est très faible : la luminosité du fond d'écran est assombrie.

\section{ETAT DE L'ART}

Notre état de l'art porte sur l'analyse de trois types de travaux : d'une part, ceux portant sur l'adaptation au service de l'accessibilité, puis les articles traitant des techniques d'adaptation sans logique floue, puis avec l'utilisation de la logique floue. En matière d'adaptation, la littérature distingue les systèmes adaptables et adaptatifs (Thevenin et al., 2003). Un système adaptable " est personnalisable sur intervention explicite de l'utilisateur qui peut agir sur les paramètres fixés par le concepteur »; un système adaptatif «est doué de discrimination. II sait reconnaître la situation (parmi plusieurs déclencheurs fixés par le concepteur) et adopte la réaction (recommandation) fixée elle aussi par le concepteur pour cette situation. Mais le système est incapable d'évaluer l'effet de sa réaction ».

Nous analysons les travaux au regard de quatre critères correspondant aux verrous présentés en introduction: la prise en charge de la multiplicité des caractéristiques des contextes d'usage (leur étendue), le traitement de l'incertitude des valeurs des caractéristiques des contextes d'usage, le niveau de complexité des règles et de la 
combinaison des règles. Les travaux sur ces trois domaines étant très nombreux, seuls les plus représentatifs du domaine sont présentés ici. Concernant le contexte, nous nous appuyons sur la définition de (Dey, 2001) : «toute information pouvant être utilisée pour caractériser la situation d'une entité. Une entité étant une personne, un endroit, ou un objet considéré comme pertinent pour l'interaction entre un utilisateur et une application, y compris l'utilisateur et les applications elles-mêmes ». Plus précisément nous étudions son étendue au regard du triplet <utilisateur, plate-forme, environnement> (Calvary et al., 2003).

Certains travaux de notre état de l'art peuvent être considérés comme relevant de plusieurs catégories. Pour faciliter la lecture, ils sont présentés dans la catégorie qui nous semble la plus pertinente. Une synthèse de tous les travaux, indépendamment de leur catégorie, est fournie en section 3.4. Dans les sous-sections qui suivent, les travaux sont décrits selon l'ordre temporel de leur parution.

\subsection{Travaux centrés sur l'accessibilité}

La notion d'accessibilité fait l'objet d'un travail de normalisation du World Wide Web Consortium (W3C) depuis la fin des années 1990, mais reste un domaine à explorer du fait de la multiplicité des situations à prendre en compte (Schmutz et al., 2017). Nous présentons ci-dessous quelques travaux que nous estimons représentatifs des différentes orientations pour l'amélioration de l'accessibilité à destination des personnes atteintes de troubles divers.

(Richards et al., 2004) présentent un mécanisme d'adaptation des pages Web qui prend en compte plusieurs aspects de l'accessibilité. Ce mécanisme s'appuie sur 13 possibilités de changement (taille de police, espacement entre les caractères, modalité vocale, suppression du multi-colonnage, effacement des images, des fonds et des animations...). II y a donc peu de règles et peu d'indications sur leur niveau de complexité. La combinaison de règles est envisagée (les utilisateurs multi-handicapés doivent pouvoir définir leurs règles ainsi que leurs combinaisons), sans plus de précision. Le contexte d'usage est réduit à quelques caractéristiques de l'utilisateur (âge, degré de handicap visuel, limitation cognitive, difficultés motrice des mains, manque d'expérience avec l'informatique), sans qu'il soit question de leur validité ou de leur incertitude.

(Kurniawan et al., 2006) présentent une liste de handicaps (visuels, moteurs, cognitifs, auditifs) liés à l'âge, déclinés en 21 caractéristiques (fatigue oculaire, réduction du champ visuel, ralentissement des réponses motrices, affaiblissement de la mémoire de travail...), avec leurs correspondances en termes d'adaptation (éviction des couleurs très soutenues, recentrage des informations critiques, remplacement des glisser-déplacer et des double-clics par d'autres interactions, ajout de rappels mémoriels...). Le contexte d'usage est donc centré sur l'utilisateur, et les règles sont à la fois simples et deux à deux exclusives : il n'y a pas de combinaison. L'incertitude n'est pas évoquée.

(Yang et Shao, 2007) proposent un système basé sur des règles d'adaptation pour prendre en compte le contexte de l'utilisateur et ses besoins. Le contexte comporte la situation de l'utilisateur (au bureau, en réunion, en train de conduire), les besoins d'accessibilité (classique, aveugle, malvoyant, sourd, malentendant) et les ressources disponibles (réseau, dispositif). Le contexte d'usage couvre donc les trois dimensions (utilisateur, plate-forme, environnement), mais de façon très partielle. L'adaptation reste relativement sommaire: les exemples fournis proposent de couper ou d'augmenter le son, d'afficher ou de supprimer les images, d'augmenter la taille de police et d'adapter la qualité audio/vidéo à la performance du réseau et à la taille de l'écran. Les règles sont donc simples et ne nécessitent pas de gestion de leur combinaison.

(Ghiani et al., 2017) ont pour but de fournir aux utilisateurs finaux un système adaptable, leur permettant de définir les règles à appliquer afin de leur permettre de personnaliser facilement et de manière autonome, le comportement de leurs applications pour l'Internet des Objets. Ils proposent un environnement permettant aux utilisateurs de spécifier facilement ces règles sous la forme de couple événement/action en limitant les conditions 
aux éléments de contexte effectivement utilisables dans la situation de l'utilisateur. Cependant, cet article ne présente pas de règles d'adaptation à proprement parler et, s'il mentionne que l'utilisateur peut définir des règles combinées à l'aide des opérateurs binaires $E T$, OU et NON, il n'est pas mentionné de moyen de définir des priorités ou des poids associés aux règles pour gérer cette combinaison.

(Kolekar et al., 2018) proposent une approche pour fournir des IHM adaptatives dans le domaine de l'apprentissage, en prenant en compte le style d'apprentissage de l'utilisateur. Les utilisateurs sont catégorisés selon 8 styles d'apprentissage (actif, réflexif, visuel, verbal...), qui induisent des choix différents dans les media et les contenus à proposer. Ainsi, un apprenant visuel se verra proposer des supports graphiques (images, graphes, vidéos), tandis qu'un apprenant verbal disposera plutôt de textes, mèls et annonces. Dans cette approche, le seul élément du contexte d'usage est le style d'apprentissage. Les règles ne peuvent pas être complexes et ne peuvent être combinées puisque les différents styles sont mutuellement exclusifs.

\subsection{Approches sans logique floue}

Cameleon (Thevenin et al., 2003) est une approche de référence, basée sur les modèles qui permet la génération dynamique d'IHM adaptée à leur contexte d'usage. II s'appuie sur un processus de réification en 4 niveaux d'abstraction : modèle de tâches, IHM abstraite, IHM concrète et enfin IHM finale. Théoriquement, toutes les caractéristiques du contexte peuvent être prises en compte. Rien ne mentionne l'incertitude de leurs valeurs. Les règles d'adaptation sont des transformations de modèles appliquées conditionnellement selon le contexte d'usage, qui doivent être deux à deux exclusives, ce qui est particulièrement complexe à obtenir, par exemple lorsque deux caractéristiques devraient induire des adaptations antagonistes.

Maria (Paternò et al., 2009) est un environnement de développement d'IHM basé sur les modèles capable de générer dynamiquement des IHM adaptées aux différentes platesformes utilisées par l'utilisateur en cas de migration d'une plate-forme à l'autre. L'article se focalise sur l'aspect plate-forme du contexte d'usage.

Supple (Gajos et al., 2010) prennent en charge la génération automatique d'IHM adaptées aux capacités, aux appareils, aux tâches, et aux préférences de chaque utilisateur. Les capacités visuelles et motrices sont les principales caractéristiques utilisateurs prises en charge lors de l'adaptation et 40 facteurs d'interface utilisateur (par exemple, la taille de police) sont considérés afin d'optimiser la disposition des éléments de l'IHM. II a été noté dans (Peissner et al., 2012) que les performances de Supple sont problématiques car elles excèdent des temps raisonnables ; cela serait donc d'autant plus vrai en cas de combinaison complexe des règles.

(Lehmann et al., 2010) définit une architecture en trois couches (3-layer architecture) pour la conception d'IHM adaptative. L'adaptation se base sur le contexte d'usage et vise les environnements intelligents. Les informations utilisées sont directement lues à partir de capteurs, sans gestion de l'incertitude. Le processus d'adaptation se limite à des « situations types ».

(Genaro Motti et al., 2012) se penchent sur l'utilisation de l'Apprentissage Automatique (Machine Learning) pour adapter des IHM au contexte d'usage, principalement dans le but d'optimiser les interactions des utilisateurs et de limiter leurs erreurs. L'Apprentissage Automatique permet de gérer des données complexes et floues, et de calculer des inférences complexes à partir de celles-ci. Cependant, l'Apprentissage Automatique nécessite de recueillir une base de connaissances importante avant de proposer des adaptations améliorant effectivement l'expérience utilisateur. De plus, cet article présente des cas d'applications potentiels, mais aucun n'a réellement été mis en pratique. 
MyUl (Peissner et al., 2012) est une infrastructure de développement d'IHM visant à améliorer l'accessibilité par le biais d'une adaptation à l'utilisateur. Les règles d'adaptation sont ici gérées grâce à des patrons. Ces travaux listent un grand nombre de caractéristiques utilisateurs. Cependant, ces caractéristiques sont principalement déclarées, et MyUI utilise des seuils fixes pour définir les différents états d'une caractéristique. En termes de règles, MyUl se focalise sur l'amélioration de l'accessibilité en fonction de certains handicaps.

Cedar (Akiki, 2016) fournit une architecture facilitant le développement d'IHM adaptatives au sein de progiciels, basé sur des simplifications en fonction de rôle. Cedar permet ainsi d'avoir une adaptation efficace, mais dans un cadre contrôlé.

(Miñon et al., 2016) propose, quant à lui, un système nommé « Adaptation Integration System », basé sur Cameleon. Ce système vise à intégrer des exigences d'accessibilité pour des personnes en situation de handicap en incluant les règles d'adaptation dans le processus de développement. Ce travail utilise des caractéristiques peu nombreuses et gère la combinaison de handicaps par la définition de sous-ensembles prédéfinis qui limitent la puissance de l'approche.

\subsection{Approches d'adaptation des IHM basées sur la logique floue}

Il existe aussi des travaux intégrant la logique floue pour la réalisation de systèmes adaptatifs.

(Beka Be Nguema et al., 2000) décrivent le développement d'une IHM tolérante à l'erreur humaine (ou HETI, pour Human-Error-Tolerant Interfaces) lors du contrôle d'un processus industriel simple. Le cas d'étude présenté est relativement simple, ne comprenant que 5 règles floues, possédant des poids, et dont la combinatoire est gérée manuellement au sein des règles. Cette contribution ne prend pas non plus en compte de caractéristiques utilisateurs, uniquement leurs actions lors du processus industriel.

(Nyongesa et al., 2003) présentent une autre approche en logique floue pour l'adaptation de page web afin d'améliorer l'expérience utilisateur. Elle se base cependant sur 7 cas d'utilisation, par exemple, Passer une commande et Effectuer une recherche. Les données sont inférées à partir des comportements utilisateurs, limitant de fait la taille du modèle utilisateur et donc les règles d'adaptation. Cette contribution n'aborde donc pas la question de la combinaison des règles qui nous semble centrale.

FSAM (Cao et al., 2005) est un outil basé sur la logique floue permettant de choisir le service le plus adapté à un utilisateur en fonction de son contexte d'usage. Ces travaux se restreignent à un choix entre des services prédéfinis et n'abordent pas les questions de l'interaction homme-machine.

(Desruelle et al., 2011) proposent une approche en logique floue pour le développement d'applications mobiles adaptatives, pour des plates-formes mobiles aux performances diverses (hardware, OS, API, etc.). L'utilisateur n'est pas pris en compte, limitant de fait le nombre de caractéristiques et leur incertitude.

(Papatheocharous et al., 2012) proposent un mécanisme d'adaptation d'IHM basé sur la logique floue, considérant trois facteurs cognitifs des utilisateurs définis par (Riding et Cheema, 1991) : «Verbal/Imager », “ Analyst/Wholist », et capacité de la mémoire de travail, chacun de ces aspects ayant des implications particulières sur le design. Ces travaux ne gèrent donc qu'un nombre restreint de caractéristiques. De plus, l'approche comprend une gestion manuelle de la composition des règles, ce qui limite les compétences du système et sa capacité à prendre en charge des situations inattendues.

(Soui et al., 2013) présentent une approche pour la personnalisation d'IHM grâce à la logique floue. Ces travaux utilisent des relations sémantiques entre composants d'IHM et préférences utilisateurs afin de sélectionner les composants les plus adaptés. Le cas d'étude présenté ne donne qu'une seule règle, à deux entrées et une sortie, et la combinatoire entre 
les entrées a été gérée manuellement au sein de la règle. Ce travail ne prend donc pas en compte des aspects qui nous semblent importants tels que l'étendue du contexte d'usage et la combinaison des règles.

Enfin, (Cueva-Fernandez et al., 2016) proposent un outil qui permet à des conducteurs ou des passagers de voiture de développer des applications au moyen d'une interface vocale. La logique floue est ici utilisée pour calculer le niveau d'expertise et de concentration de l'utilisateur : débutant, intermédiaire ou avancé, chaque niveau impliquant la fourniture de plus ou moins d'options et d'informations. Les règles floues utilisées sont au nombre de 7 . Elles utilisent 10 caractéristiques (nombre d'applications créées ; nombre de demandes d'aide ; nombre de jurons...) afin de modifier une sortie : le niveau d'expertise. Cette utilisation de la logique floue ne correspond donc pas à ce que nous souhaitons faire en termes de règle d'adaptation.

\section{$3.4 \quad$ Analyse}

Notre analyse des travaux existants s'appuie sur des critères relatifs aux difficultés de réalisation de moteur d'adaptation. II s'agit de : 1) l'étendue du contexte d'usage pris en compte en termes de couverture des 3 aspects (utilisateur, plate-forme, environnement) ainsi que du nombre de caractéristiques considérées, 2) la gestion de l'incertitude sur les valeurs des caractéristiques du contexte d'usage, 3) la complexité et 4) la combinaison des règles d'adaptation.

Le tableau 1 regroupe les différents travaux cités ci-dessus et les évalue au regard de nos critères avec des boules de Harvey. Les critères considérés permettent de regrouper les différents travaux présentés dans les sous-sections précédentes.

Les travaux centrés sur l'accessibilité et décrits en section 3.1 (Richards et al., 2004 ; Kurniawan et al., 2006 ; Yang et Shao, 2007 ; Ghiani et al., 2017 ; Kolekar et al., 2018) se focalisent sur l'utilisateur pour le calcul des adaptations, sans toutefois prendre en compte un nombre important de caractéristiques. La prise en compte de l'étendue du contexte d'usage peut donc être considérée comme limitée. En matière d'adaptation, il est parfois difficile de savoir si ces approches sont statiques, adaptatives ou adaptables. Enfin, l'incertitude, la complexité ou la combinaison des règles ne sont que rarement mentionnées et ne sont pas traitées dans les travaux sur l'accessibilité.

Les travaux (Thevenin et al., 2003 ; Paternò et al., 2009 ; Gajos et al., 2010 ; Lehmann et al., 2010 ; Genaro Motti et al. 2012 ; Peissner et al., 2012 ; Akiki, 2016 ; Miñon et al., 2016), décrits en section 3.2, traitent tous d'adaptation dynamique en fonction d'un contexte d'usage plus ou moins étendu et comprenant un nombre plus ou moins élevé de caractéristiques. Ces travaux, à l'exception de (Genaro Motti et al., 2012) détaillé page suivante, s'appuient sur l'Ingénierie Dirigée par les Modèles. Cependant, selon (Akiki et al., 2014), I'IDM ne parvient pas à produire des IHM adaptables jusqu'aux utilisateurs finaux. En effet, les approches IDM souffrent de plusieurs limites : (1) le paradigme, qui repose sur des modèles et des langages complexes, est difficile à apprendre (Marçal de Oliveira et al., 2015) et encore plus à maîtriser par les développeurs (Myers et al., 2000 ; Abrahao et al., 2017). Les développeurs ont des difficultés à produire les " bons modèles à chaque niveau d'abstraction » (Lütjen et al., 2014). De plus, I'IDM ne parvient pas non plus à garantir que le système résultant sera fonctionnel (Mussbacher et al., 2014); (2) lorsqu'ils adoptent une approche basée sur les modèles, les développeurs doivent produire tous les modèles et transformations nécessaires avant de générer les interfaces utilisateur, ce qui conduit à une sorte d'effet tunnel (Deuff et al., 2013) ; (3) la génération d'IHM par l'IDM n'est pas suffisamment mature pour couvrir tous les besoins des utilisateurs finaux, et notamment la capacité de basculer entre modalités (Coutaz, 2010) ; (4) la convivialité des interfaces utilisateur générées par les approches IDM est généralement considérée comme faible (Meixner et al., 2011) ; de plus, les modèles étant complexes, leur utilisation est réservée à des experts, ce qui empêche la participation des utilisateurs finaux au processus de 
Tableau 1. Comparaison des travaux de l'Etat de l'Art en fonction des critères ciblés

\begin{tabular}{|c|c|c|c|c|}
\hline $\begin{array}{l}\text { d } \\
\frac{1}{0} \\
\frac{0}{0} \\
\frac{0}{2}\end{array}$ & 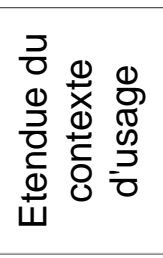 & 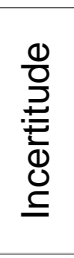 & 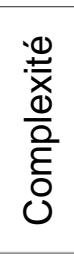 & 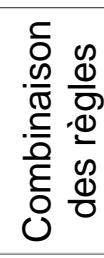 \\
\hline (Richards et al., 2004) & 1 & 0 & 0 & 0 \\
\hline (Kurniawan et al., 2006) & 1 & 0 & 0 & 0 \\
\hline (Yang et Shao, 2007) & 1 & $\bigcirc$ & $\bigcirc$ & $\bigcirc$ \\
\hline (Ghiani et al., 2017) & 1 & 0 & 0 & 0 \\
\hline (Kolekar et al., 2018) & 1 & 0 & 0 & 0 \\
\hline Cameleon (Thevenin et al., 2003) & ○ & 0 & 0 & 1 \\
\hline Maria (Paternò et al., 2009) & 1 & 0 & 0 & 0 \\
\hline Supple (Gajos et al., 2010) & 1 & ○ & O & O \\
\hline 3-layer architect (Lehmann et al., 2010) & 0 & 0 & 0 & 0 \\
\hline MyUI (Peissner et al., 2012) & ○ & 0 & O & 1 \\
\hline Cedar (Akiki, 2016) & 1 & ○ & O & O \\
\hline (Miñon et al., 2016) & ? & 0 & O & 1 \\
\hline (Genaro Motti et al., 2012) & 0 & - & 0 & 0 \\
\hline (Beka Be Nguema et al., 2000) & 0 & 0 & 0 & 0 \\
\hline (Nyongesa et al., 2003) & 1 & $\bullet$ & 0 & ○ \\
\hline FSAM (Cao et al., 2005) & 1 & - & 0 & 1 \\
\hline (Desruelle et al., 2011) & $\bigcirc$ & 0 & $\bigcirc$ & 1 \\
\hline (Papatheocharous et al., 2012) & 0 & 0 & 0 & 0 \\
\hline (Soui et al., 2013) & 0 & - & 0 & O \\
\hline (Cueva-Fernandez et al., 2016) & 0 & 0 & 0 & 0 \\
\hline
\end{tabular}

: traité, 1 : partiellement traité, $\bigcirc$ : non traité

développement et bloque ainsi l'utilisation des approches centrées utilisateurs, recommandées pour assurer leur satisfaction (Akiki et al., 2014 ; Abrahao et al., 2017). Par conséquent, il est difficile d'atteindre les attentes utilisateurs en termes de facilité d'utilisation, d'apprentissage, de satisfaction, d'attractivité, les affects ou les émotions induits - en bref, une bonne expérience utilisateur (Abrahao et al., 2017 ; ISO 9241-210:2010 ; Law et al., 2009). De manière générale, les travaux sur l'adaptation au contexte ne se penchent pas sur l'incertitude, la complexité ou la combinaison des règles.

Les travaux de (Beka Be Nguema et al., 2000 ; Nyongesa et al., 2003 ; Cao et al., 2005 ; Desruelle et al., 2011 ; Papatheocharous et al., 2012 ; Soui et al., 2013 ; Cueva-Fernandez et al., 2016) utilisent la logique floue et prennent bien en compte l'incertitude des valeurs. Dans les travaux de (Cao et al., 2005 ; Desruelle et al., 2011), la composition des règles est gérée par la logique floue, mais l'absence de prise en compte d'un contexte d'usage à large couverture limite les capacités de la solution. Aucun de ces travaux n'aborde vraiment la question de la combinaison des règles.

Enfin, les travaux de Genaro Motti (Genaro Motti et al., 2012) ont les meilleures notes globales, gérant 3 de nos 4 critères. Cependant, l'utilisation de l'Apprentissage Automatique 
nécessite de disposer d'une base de connaissances importante - et donc difficile à acquérir - avant de pouvoir effectuer des adaptations correspondant effectivement aux besoins des utilisateurs.

Afin de dépasser les limites des travaux actuels et de proposer une solution qui permette de gérer l'incertitude du contexte et de nombreuses caractéristiques et règles d'adaptation, nous nous intéressons à la réalisation d'un système d'adaptation des IHM basé sur la logique floue. Pour aller au-delà des propositions existantes en logique floue, nous montrerons son intérêt pour deux aspects peu abordés jusqu'alors qui sont la complexité des règles et la combinaison des règles d'adaptation.

\section{LOGIQUE FLOUE}

Afin de comprendre l'utilisation de la logique floue pour l'adaptation des IHM, cette section en présente les principes et son processus d'inférence.

\subsection{Systèmes experts flous}

Les systèmes experts flous sont des algorithmes d'intelligence artificielle capables de reproduire des raisonnements à partir de règles basées sur la logique floue. La logique floue peut être vue comme une extension de la logique booléenne classique pour prendre en compte l'incertitude et l'imprécision : alors que la logique classique ne considère que deux valeurs (《vrai » et « faux »), la logique floue considère un ensemble infini de valeurs réelles comprises entre 0 et 1 . Cette logique a été introduite par Zadeh (Zadeh, 1965) afin de se rapprocher du raisonnement humain et d'éviter les effets de seuil des opérateurs de comparaison. La logique floue permet également d'écrire des règles plus proches du langage naturel.

\subsection{Concepts de sous-ensemble flou et de variable linguistique}

La logique floue est basée sur le concept de sous-ensemble flou. Soit $X$ l'univers du discours, c'est-à-dire un ensemble d'objets dénotés $x$. Un sous-ensemble flou $A$ de $X$ est totalement caractérisé par une fonction appelée "fonction d'appartenance ». Cette fonction indique à quel point un objet $x$ appartient à $X$, alors que dans les ensembles « crisp » (le terme " crisp » se définit par opposition à flou), les objets appartiennent ou non à l'ensemble. Les sous-ensembles flous sont donc utiles pour représenter l'imprécision des entrées ou l'incertitude liée aux connaissances mises en œuvre. La figure 1 représente un sousensemble flou défini sur [0;10] avec une fonction d'appartenance triangulaire qui représente « approximativement 5 ».

Figure 1. Exemple de sous-ensemble flou

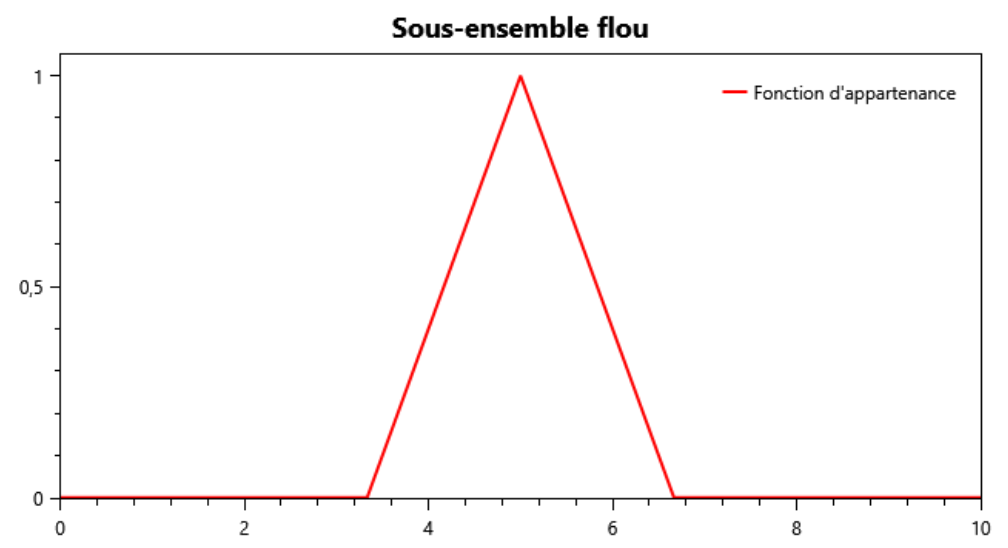

Les fonctions d'appartenance peuvent prendre différentes formes: triangulaire, trapézoïdale, Gaussienne, etc. 
Le concept suivant dérive des sous-ensembles flous et s'appelle " variable linguistique » (Zadeh, 1975) qui est un triplet $\left(\mathrm{V}, \mathrm{X}_{\mathrm{V}}, \mathrm{T}_{\mathrm{V}}\right.$ ) défini par : 1) $\mathrm{V}$, son nom ; 2) $\mathrm{X}_{\mathrm{V}}$, le domaine sur lequel elle est définie ; 3) $\mathrm{T}_{\mathrm{v}}$, une collection finie de sous-ensembles flous appelés « termes » qui qualifient $V$ et qui sont nommés.

La figure 2 représente une variable linguistique appelée « Acuité visuelle » définie sur le domaine [0;4] (similairement aux propositions de MyUI (Peissner et al., 2012)), composée de quatre termes («Normal » , «Faible », «Très faible » et « Minimal ») et leurs fonctions d'appartenance associées.

Figure 2. Exemple de variable linguistique : Acuité visuelle

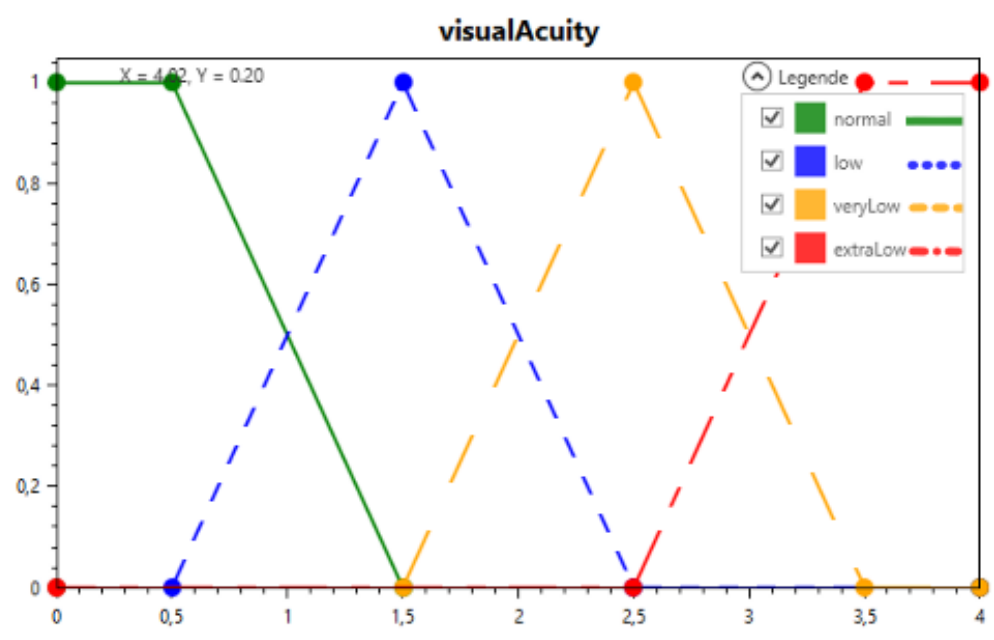

\subsection{Utilisation de règles floues pour modéliser les connaissances}

Les définitions précédentes sont utilisées pour définir les connaissances d'experts humains dans un ensemble de règles. Ces règles sont basées sur une combinaison de propositions floues élémentaires. Une proposition floue élémentaire est la définition de « $V$ est $A$ » à partir d'une variable linguistique $\left(V, X_{V}, T_{V}\right)$ où $A$ est un terme de $T_{V}$. Ainsi, si l'on reprend la variable linguistique "Acuité visuelle" de la figure 2, une proposition floue pourrait être «Acuité visuelle est faible ». Cette proposition est évaluée à partir de la fonction d'appartenance du terme impliqué pour une acuité visuelle donnée. Cette valeur est floue et donc comprise entre $[0,1]$ contrairement à la logique classique dans laquelle l'évaluation d'une proposition est soit vraie (1) soit fausse (0).

Une expression floue est une composition de propositions floues élémentaires ou d'autres expressions à l'aide d'opérateurs logiques: négation (non), conjonction (et), disjonction (ou), le ou exclusif n'étant pas géré.

Les propositions floues sont des cas particuliers d'expressions floues. Par exemple, soit deux propositions floues « $V$ est $A$ » utilisant $\left(V, X_{v}, T_{V}\right)$ et « $W$ est $B$ » utilisant $\left(W, X_{W}, T_{W}\right)$. Alors, " $V$ est $A$ et $W$ est $B$ », " $V$ est $A$ ou non $W$ est $B$ » sont des expressions floues. La valeur de vérité des expressions floues est obtenue en appliquant les opérateurs sur les valeurs de vérité de leurs opérandes.

Ces expressions sont donc utilisées pour exprimer les connaissances des experts humains dans les règles floues. Une règle floue est composée d'une prémisse (ou antécédent) et d'une conclusion (ou conséquence) et est de la forme "SI prémisse ALORS conclusion". Une prémisse est une expression floue alors qu'une conclusion est une déclaration floue de nature différente. Une conclusion peut être une proposition floue. Dans ce cas, la règle est de la forme : «SI Acuité visuelle est faible ALORS la taille de police est élevée. " Une conclusion peut être une fonction mathématique des entrées de la prémisse. Ces règles sont dites de la forme Takagi-Sugeno et sont de la forme "SI acuité visuelle est faible ALORS taille_de_police=f(acuité visuelle) ». 
Ainsi, les règles associent les entrées du système aux sorties à l'aide d'une implication floue (dénotée par ALORS).

\subsection{Inférence floue}

Le formalisme utilisé pour représenter les connaissances ayant été présenté, nous pouvons nous intéresser au processus d'inférence, c'est-à-dire au calcul des sorties. En effet, dans la section précédente nous avons vu comment une règle fonctionnait ; nous allons à présent voir comment les règles fonctionnent ensemble. II existe deux types d'inférence en logique floue en fonction du type de règle utilisé : l'inférence de Mamdani et l'inférence de Takagi-Sugeno. Dans les deux cas, les règles sont évaluées individuellement puis agrégées en utilisant leur activation pour pondérer leurs sorties respectives et obtenir ainsi la valeur de sortie finale. De par la nature de leurs conclusions, les systèmes de Mamdani sont plus interprétables et l'écriture des règles est plus intuitive, contrairement aux systèmes de Sugeno qui sont plus appropriés à l'apprentissage automatique. Nos règles provenant d'une expertise, nous avons naturellement choisi un système de Mamdani (Bouchon-Meunier, 2007) dont le processus d'inférence est décrit ci-après et résumé dans la figure 3.

Figure 3. Vue d'ensemble de l'inférence de Mamdani

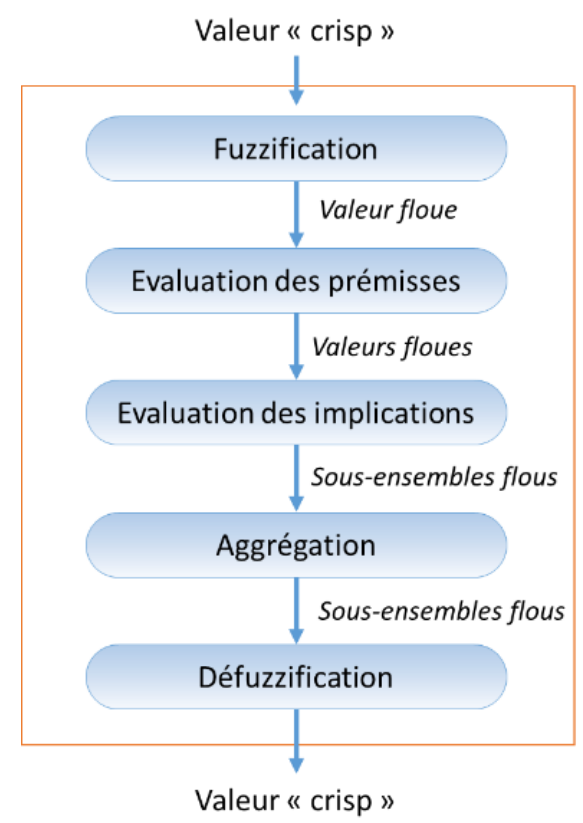

La première étape consiste à évaluer chacune des propositions floues élémentaires présentes dans les prémisses des règles : cette étape est appelée fuzzification. Elle prend les valeurs "crisp " des entrées et associe une valeur floue en fonction des fonctions d'appartenance des termes. Par exemple, en utilisant la variable linguistique "Acuité visuelle » de la figure 2 et le terme "normal », pour une acuité visuelle de 0 , " acuité visuelle est normale » est évaluée à 1. Pour une acuité visuelle de 1, "acuité visuelle est normale » et « acuité visuelle est faible » sont chacun évalués à 0.5 .

Les prémisses sont ensuite calculées. La valeur des prémisses devient alors la valeur d'activation de la règle. Par exemple, si on prend les opérateurs de Zadeh, pour une acuité visuelle de 0 , la valeur de la prémisse "acuité visuelle est normale ou acuité visuelle est faible » est $\max (1,0)=1$.

La fonction d'implication est ensuite appliquée à la conclusion des règles. Le résultat est un sous-ensemble flou qui peut être interprété comme une distribution des valeurs pour les sorties. La figure 4 montre le sous-ensemble flou résultant de l'application d'une implication minimum lorsque la règle est activée à 0,75 . 
Les sous-ensembles flous des différentes règles concernant la même sortie sont ensuite agrégés par une méthode particulière, comme par exemple la fonction maximum (figure 5).

Enfin, le sous-ensemble flou de chaque sortie est transformé en valeur «crisp » : cette étape est appelée défuzzification. II existe différentes fonctions de défuzzification. La plus connue consiste à calculer le barycentre de l'aire sous la courbe (figure 5).

Figure 4. Résultat de l'implication

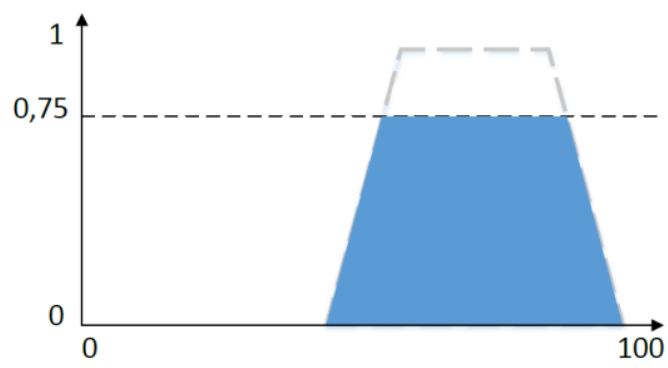

Figure 5. Application de l'agrégation maximum à deux sous-ensembles flous

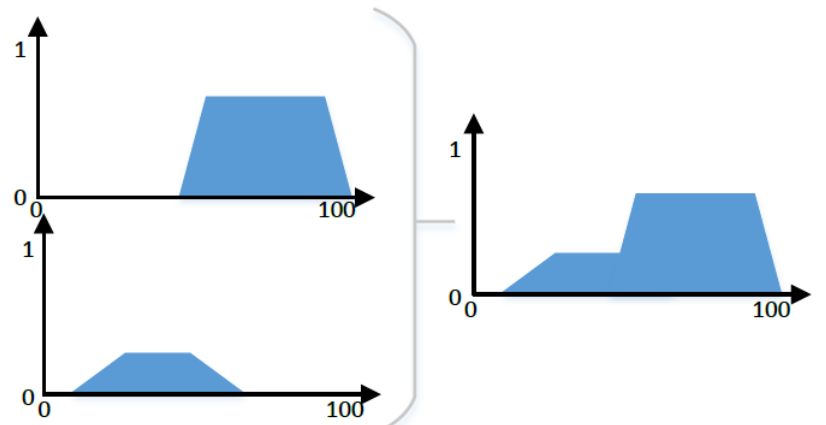

Pour nos travaux, nous avons choisi de remplacer l'étape d'agrégation et de défuzzification par une étape unique qui consiste à calculer le barycentre de chacune des sorties individuellement et de les pondérer entre eux en fonction de l'aire obtenue par l'implication floue. Ce changement a été effectué car il a pu être adapté aux domaines discrets ordonnés : la valeur la plus proche de la somme pondérée des barycentres est alors utilisée comme valeur finale. Cela permet d'assurer une transition progressive entre les différentes valeurs d'une sortie, même dans le cas de domaines discrets ordonnés.

\section{LE SYSTEME FUZZY4U}

Dans cette section, nous montrons les apports de la logique floue au sein d'un système d'adaptation des IHM, en particulier pour l'accessibilité. Nous commençons par présenter l'architecture logicielle de Fuzzy4u avant de discuter de l'intégration de la logique floue dans son moteur d'adaptation.

\subsection{Architecture logicielle}

L'architecture de Fuzzy4U comprend 3 composants principaux : un moteur d'inférence, un moteur d'adaptation et le système interactif à adapter (voir figure 6).

Le moteur d'inférence a la charge de déduire dynamiquement la valeur du contexte d'usage à partir des données captées, par exemple, le nombre de clics «valides » effectués sur des interacteurs tels que liens, boutons, listes, et le nombre de clics « invalides » (à côté de tout interacteur) afin de déterminer si l'utilisateur a une bonne précision de la main.

D'autres données peuvent être déclarées par l'utilisateur au moyen d'une méta-UI (Coutaz, 2006). Dans notre cas, cette méta-IHM regroupe un ensemble de caractéristiques que l'utilisateur peut manipuler pour contrôler l'adaptation, telles que ses caractéristiques 
(dyslexie, daltonisme, acuité visuelle, etc.) ou encore directement des caractéristiques de l'IHM (taille de police, espacement des mots, etc.). Notre méta-IHM se limite ici aux caractéristiques de l'utilisateur. Elle ne permet pas de visualiser ou de modifier les règles d'adaptation.

Les données inférées et déclarées sont envoyées au moteur d'adaptation. En cas de données inférées et déclarées portant sur la même caractéristique, ce seront les données fixées par l'utilisateur (donc déclarées) qui seront prises en compte.

Le moteur d'adaptation se sert ensuite des données ainsi que d'une base de règles d'adaptation pour sélectionner une version adéquate de l'IHM, appelée variant (Céret et al., 2016), sur laquelle des paramètres de présentation (couleurs, police de caractères...) seront appliqués. Par exemple, si la précision de la main de l'utilisateur est faible, le moteur d'adaptation peut augmenter l'espace pris en compte pour le clic sur les interacteurs afin d'améliorer l'utilisabilité de l'interface pour cet utilisateur spécifique.

Figure 6. Architecture logicielle de Fuzzy4U pour l'adaptation d'IHM

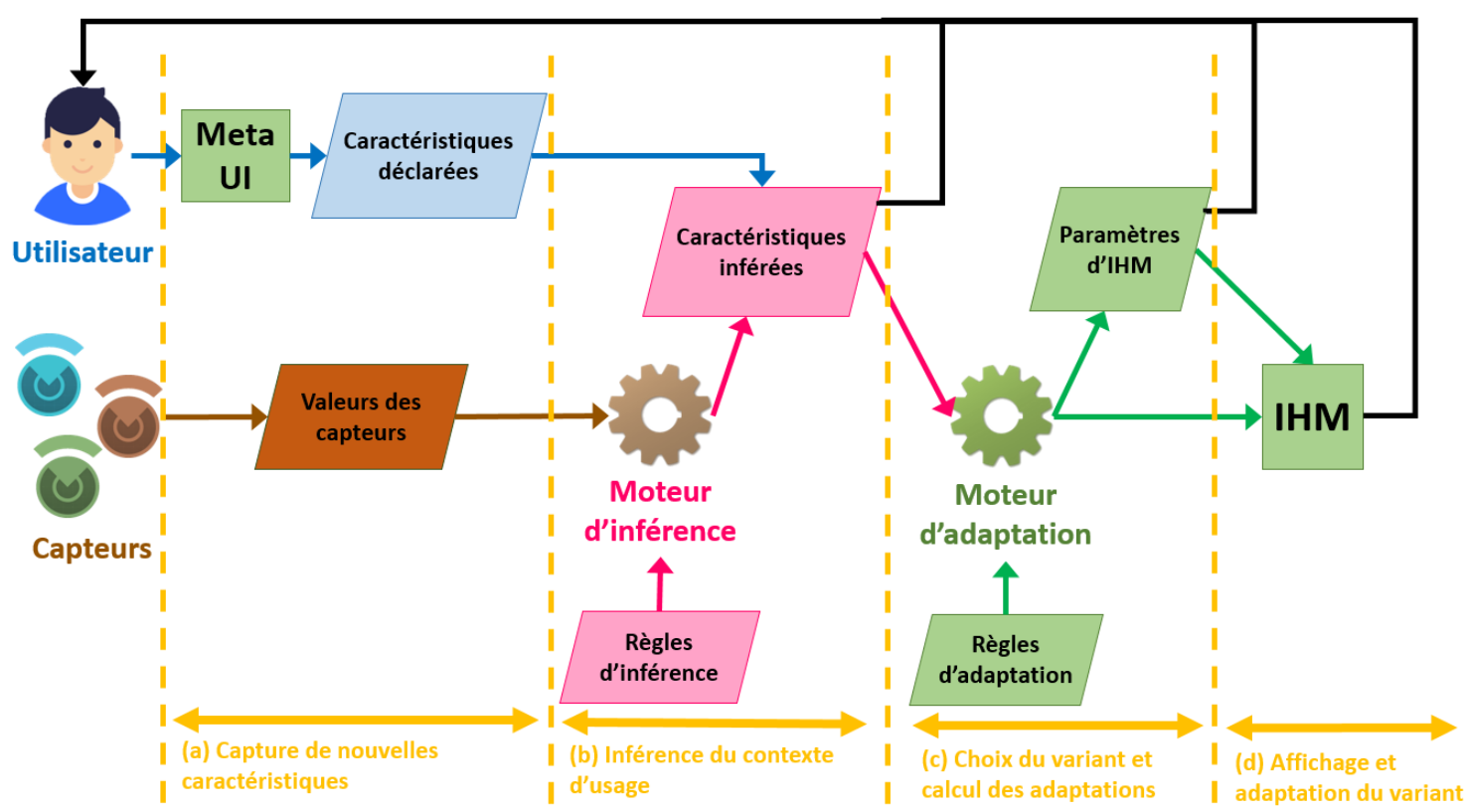

Nous illustrons le fonctionnement de Fuzzy4U sur l'exemple fil rouge. Les capteurs de luminosité ambiante perçue par la plate-forme (par exemple 50.000 lux) ainsi que le nombre de bons et de mauvais clics (par exemple, 130 clics sur un interacteur et 212 dans une zone vide) servent d'entrées initiales. Le moteur d'inférence, selon sa base de règles, transforme les « 50.000 lux » en «luminosité : plein soleil », ainsi que les clics en «précision de la main : très faible ».

Cependant, l'utilisateur, Bernard, a pu utiliser la méta-IHM pour déclarer certaines caractéristiques, telles que la précision des mains qu'il a déclarée comme étant « bonne ». Etant donné qu'une caractéristique a été à la fois inférée et déclarée, la valeur déclarée prend la priorité, et la précision des mains connue par le système pour Bernard restera donc «bonne».

Ces deux caractéristiques (bonne précision de la main et luminosité plein soleil) sont ensuite transmises au moteur d'adaptation qui détermine si des adaptations sont nécessaires. En l'occurrence, une bonne précision des mains n'implique pas de modification de l'IHM, mais l'éclairage ambiant entraîne une augmentation du contraste et de la luminosité afin de conserver une lisibilité suffisante de l'écran.

Le moteur d'inférence est distinct du moteur d'adaptation dans un but de séparation des préoccupations : le premier est chargé de transformer des valeurs brutes (comme un certain 
nombre de clics valides et invalides) en valeurs utilisables par le second (comme une précision de la main « très faible »).

\subsection{Les apports de la logique floue}

\subsubsection{Incertitude}

Toutes les caractéristiques ont un degré d'incertitude, qu'elles soient inférées par le moteur (ex: imprécision du calcul de la précision des mains grâce à un ratio entre clics valides et clics invalides) ou déclarées par l'utilisateur (ex : deux utilisateurs ayant une acuité visuelle identique pourraient chacun la définir différemment).

La logique floue permet, grâce aux fonctions d'appartenance (voir la définition en section 3 ) de prendre en compte une incertitude. Par exemple, la figure 2 montre la définition de la variable linguistique (voir la définition en section 3) acuité visuelle : la valeur est définie en abscisses (ici de 0 à 4), et la certitude des 4 différents états (normal, low, veryLow et extraLow) est spécifiée en ordonnées. L'état « normal » est ainsi certain lorsque que l'acuité visuelle varie de 0 à 0.5 . À partir de 0.5 et jusqu'à 1.5 , la certitude de l'état « normal » décroît au profit de l'état «low » qui gagne en certitude. À 1.5, l'état «low » a donc 100\% de certitude alors que l'état « normal » est à $0 \%$. Le croisement des courbes (aux abscisses 1, 2 et 3 ) indique donc une probabilité de $50 \%$ pour les deux états.

\subsubsection{Complexité des règles}

Outre la prise en compte de l'incertitude, des difficultés sont liées à l'expression des règles d'adaptation. En particulier, les règles qui disposent de nombreux seuils peuvent devenir assez lourdes en logique booléenne.

Reprenons l'exemple de la règle sur le niveau d'acuité visuelle présenté en section 4.2. Nous l'avons vu, plusieurs seuils sont nécessaires (ici, 1, 2, 3 et 4) et la granularité finale de la variable de sortie est relativement faible (ici, 4 valeurs possibles : 12, 14, 16, 18). Cette granularité peut être améliorée, mais au détriment du nombre de seuils.

En logique floue, l'implémentation de cette règle comprend la définition des variables linguistiques d'entrée et de sortie (figure 7 ), et la création des règles en logique floue (figure 8).

Figure 7. Définition des variables linguistiques d'acuité visuelle et de taille de police de caractères

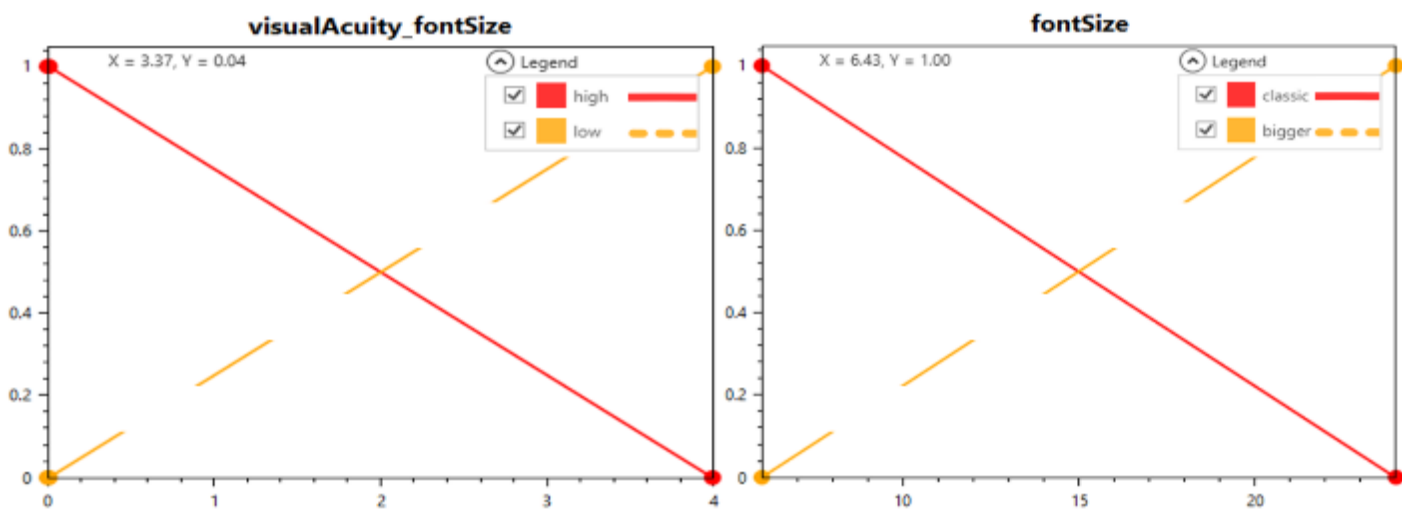

Figure 8. Définition de règles floues pour le calcul de la taille de police en fonction de l'acuité visuelle

If visualAcuity_fontSize is high then fontSize is classic
If visualAcuity_fontSize is low then fontSize is bigger


Compte tenu des définitions des domaines de variables, la règle en logique floue est simple et ne comporte que deux cas.

En utilisant la méthode de défuzzification des barycentres pondérés (ou centre de gravité), la valeur de sortie fontSize est comprise entre 12 et 18 points. Son principe est de calculer le barycentre du sous-ensemble flou de sortie pour chacune des règles, et d'en faire une somme pondérée par l'aire sous la courbe. En considérant toutes les règles ayant une même sortie, la valeur défuzzifiée peut s'écrire :

$$
d(\text { sortie })=\frac{\sum_{i} C_{i} \times A_{i}}{\sum_{i} A_{i}}
$$

où $C_{i}$ est la valeur du barycentre et $A_{i}$ l'aire sous la courbe pour la ième règle concernant cette sortie.

Cette défuzzification est utilisable sur des variables linguistiques continues. Nous l'avons adaptée aux variables discrètes car, dans notre cas, les domaines discrets sont ordonnés, ce qui nous permet de donner du sens au barycentre. Dans ce cas, l'aire sous la courbe est simplement remplacée par la valeur correspondante dans l'histogramme qui sert de fonction d'appartenance.

Afin de comprendre le principe de cette méthode, deux exemples sont donnés dans les figures 9 et 10 .

Sur la figure 9, on voit que pour une acuité visuelle à 0 en entrée, le terme « high » est activé à $100 \%$ et le terme "low » à $0 \%$. Selon la première règle, l'activation du terme de sortie "classic » se fait lui aussi à 100\%, représenté par l'aire rouge. Le barycentre de ce triangle rectangle est situé au premier tiers de sa base, soit une valeur de 12 pour la variable de sortie fontSize ayant un domaine entre 6 et 24 .

Figure 9. Schéma explicatif de la méthode de défuzzification des barycentres pondérés pour une valeur d'entrée de 0

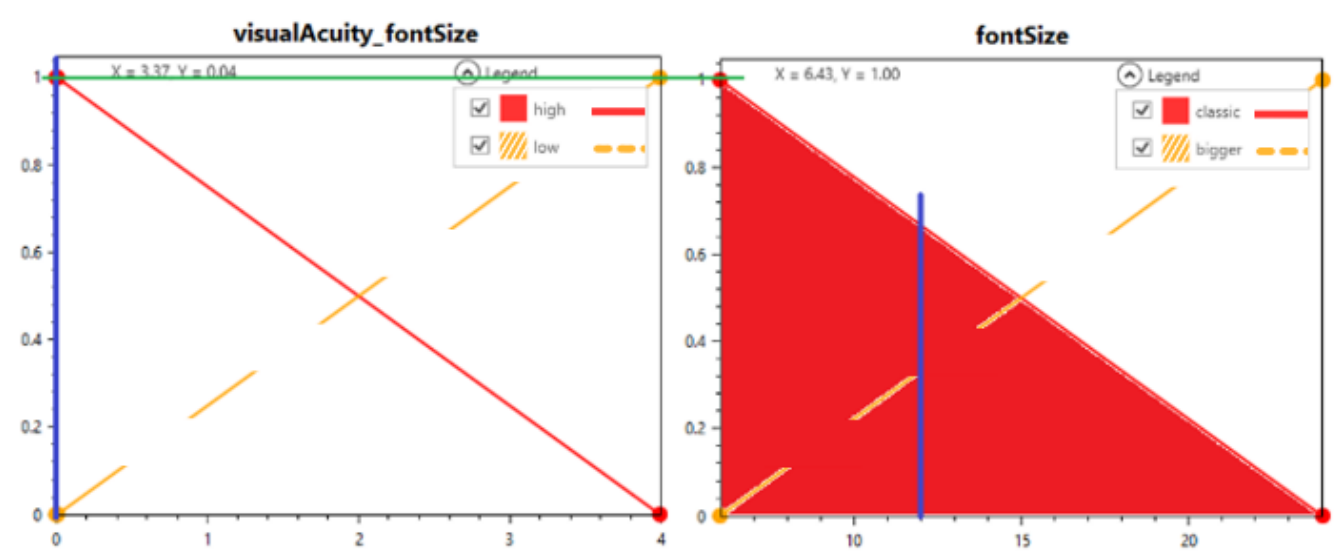

Pour une acuité visuelle à 1 en entrée (figure 10), le terme « high » est cette fois activé à $75 \%$ et le terme « low » à $25 \%$. Cela est représenté par les deux lignes vertes et l'activation du terme de sortie "classic » à 75\%, représenté par l'aire rouge et l'activation du terme de sortie "bigger » à 25\%, représenté par l'aire orange. Les barycentres de ces deux quadrilatères sont ensuite calculés (12.3 pour le barycentre lié à " classic » et 10.071 pour celui lié à «bigger »), puis pondérés en fonction de leurs aires (respectivement 84.375 et 39.375), afin d'obtenir le résultat final de 13.5 points pour la variable fontSize. Nous avons ainsi l'état " bigger » qui a pris un peu plus de poids comparé à l'état " classic ", donnant une taille de police de 13.5 au lieu de la valeur 12 du cas précédent. Ces résultats sont donc continus et suivent une fonction « fontSize $=12+$ visualAcuity * 1.5 ». 
Figure 10. Schéma explicatif de la méthode de défuzzification des barycentres pondérés pour une valeur d'entrée de 1

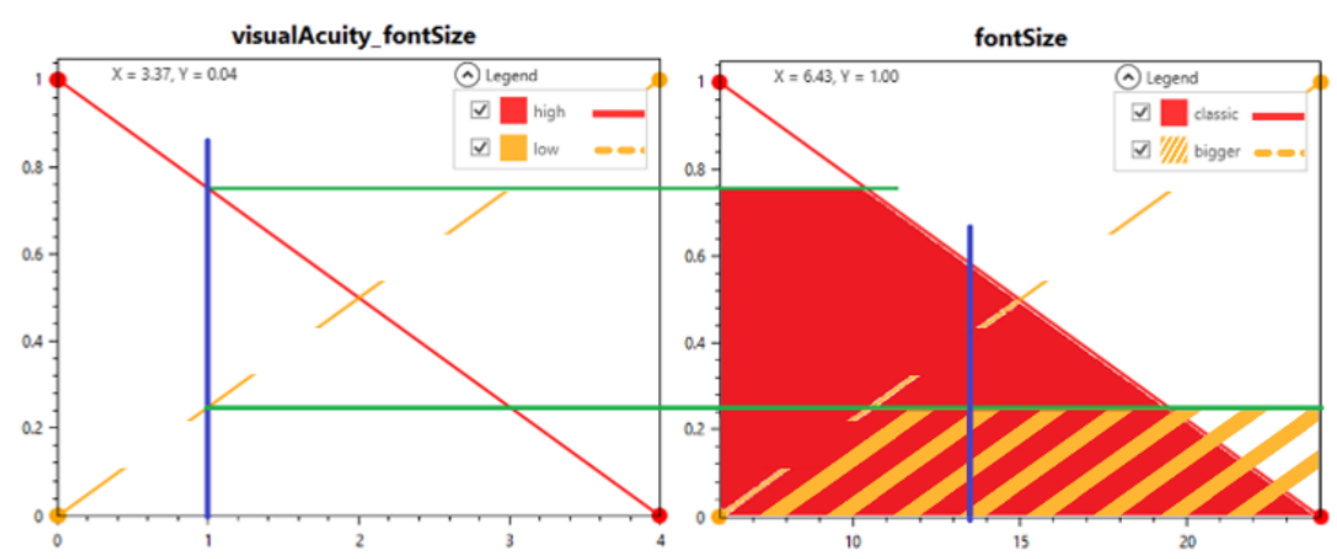

Cet exemple montre comment la logique floue a les capacités d'éviter les effets de seuil. Elle permet dans certains cas de simplifier l'expression des règles d'adaptation tout en permettant d'obtenir des valeurs de sortie plus précises.

\subsubsection{Combinaison de règles}

Le dernier verrou à aborder est la combinatoire des règles qui peut survenir lorsque plusieurs variables d'entrées peuvent influer sur une même variable de sortie (Blouin et al., 2011). Par exemple, il peut être nécessaire d'augmenter l'espace cliquable autour des interacteurs, soit quand l'acuité visuelle est faible, soit quand la précision des clics (et donc des mains) est faible. Un problème se pose quand l'une des deux variables d'entrée est forte alors que l'autre est faible.

La logique floue a l'avantage de pouvoir gérer cela de manière intrinsèque, notamment par le biais de la gestion de l'incertitude : sans avoir un poids différent, une certitude plus importante a plus d'impact. II reste toutefois possible de spécifier des poids au besoin.

Considérons l'exemple de la taille de la zone de clic des interacteurs, nommée ici clickCorrection. Elle se base sur l'acuité visuelle (figure 2) et la précision des mains (dont la forme du graphique est identique à celui de la figure 2) afin de définir un niveau plus ou moins important de correction de la taille ; la variable linguistique de sortie, clickCorrection, étant composée de 4 valeurs discrètes représentant 4 degrés de correction allant de noCorrection à highCorrection (figure 11).

Figure 11. Règles floues définies pour le calcul du clickCorrection en fonction de la précision des mains et de l'acuité visuelle

If handPrecision is normal then clickCorrection is noCorrection
If handPrecision is low then clickCorrection is lowCorrection
If handPrecision is veryLow then clickCorrection is mediumCorrection
If handPrecision is extraLow then clickCorrection is highCorrection
If visualAcuity is normal then clickCorrection is noCorrection
If visualAcuity is low then clickCorrection is lowCorrection
If visualAcuity is veryLow then clickCorrection is mediumCorrection
If visualAcuity is extraLow then clickCorrection is highCorrection

Les 4 premières règles se basent sur l'état de la variable handPrecision - normal, low, veryLow, extraLow - et fournissent une valeur de sortie pour clickCorrection - allant 
respectivement de noCorrection à highCorrection. Les 4 dernières règles sont identiques à l'exception de la variable d'entrée qui est cette fois visualAcuity. On peut noter qu'il n'est pas nécessaire dans l'expression de ces règles de se soucier de leur éventuelle contradiction ou de leur combinaison. En logique floue, la valeur de sortie est déterminée par la défuzzification.

Dans l'exemple de la correction de la taille des interacteurs pour obtenir une transition lissée d'une valeur à l'autre des paramètres de sortie, nous avons opté pour la défuzzification dite des barycentres pondérés (cf. sous-section 4.2.2). Ainsi, avec une acuité visuelle à 0 et une précision des mains à 4 en entrée, le résultat est « lowCorrection ». Alors qu'une acuité visuelle à 1 et une précision des mains à 4 en entrée donneront une sortie «mediumCorrection ».

Cet exemple montre comment la logique floue permet de gérer la combinaison des règles d'adaptation par la défuzzification, sans avoir à mettre en place un algorithme spécifique de priorisation.

Maintenant que nous avons décrit des avantages de la logique floue, la prochaine partie détaillera comment des recommandations d'accessibilité peuvent être traduites en règles floues.

\section{REGLES POUR L’ACCESSIBILITE}

Le premier point à noter est que certaines préconisations pour l'accessibilité sont destinées à être exploitées lors de la création de l'IHM par un concepteur ou un développeur humain et sont difficiles, en l'état actuel, à implémenter sous forme d'adaptation d'IHM. Par exemple, la règle 1.2.1 du WCAG précise que tout contenu purement vidéo doit être accompagné a minima soit d'une auto-description, soit d'une version alternative (textuelle ou audio). La mise à disposition d'une telle alternative ne relève pas du système d'adaptation. On pourrait dès lors supposer que la règle 1.2.1 n'est pas utile pour notre objectif. Cependant, si l'on va plus loin, on peut réinterpréter la règle en termes d'adaptation : lorsque l'utilisateur est malvoyant et que l'interface propose un support purement visuel, il convient d'offrir par défaut une version alternative, comme l'affichage en braille de l'auto-description si l'utilisateur dispose de cette possibilité, ou l'utilisation d'une version audio. Lorsque l'utilisateur est sourd, il convient d'afficher les sous-titres sans que l'utilisateur ait à les demander.

Nous présentons ci-dessous la traduction en règles des trois catégories de recommandations citées (WCAG, EAPM et WAI-AGE). Les règles sont dans un premier temps exprimées suivant la syntaxe la plus communément utilisée, "If ... then... else... », avant de montrer comment elles sont exploitées dans notre proposition. Notre objectif ici est d'obtenir des règles aussi simples que possible, quitte à en formuler plusieurs, éventuellement contradictoires. L'intérêt principal de cette approche est d'obtenir des règles facilement compréhensibles et facilement réalisables.

De plus, nos règles sont structurées pour prendre en compte la situation de l'utilisateur à chaque instant, et ainsi adapter les IHM aux difficultés, qu'elles soient persistantes ou situationnelles (Wobbrock, 2019). Pour cela, elles s'appuient sur des caractéristiques dynamiques et évolutives du contexte d'usage. Ces caractéristiques peuvent être, soit déclarées par l'utilisateur, soit, lorsque c'est possible, inférées par le système. Par exemple, on peut inférer la tranche d'âge d'une personne par l'analyse d'une photographie prise avec la caméra de son dispositif, mais ses préférences en termes de couleurs ou de volumétrie de texte doivent être déclarées. D'autre part, les caractéristiques peuvent être, soit énumérées (la tranche d'âge peut être 'kid', 'teenager', 'grown-up', ou 'senior'), soit être plus ou moins vraies (la dyslexie pouvant être plus ou moins prononcée, on considère que l'attribut 'dyslexic' est plus ou moins vrai). Les règles sont destinées à donner des indications sur les caractéristiques de l'IHM, par exemple, avoir comme résultat 'language=FR' (la langue 
utilisée dans l'interface est le français) ou 'backgroundLuminosity is darker' (l'arrière-plan doit être assombri).

Dans les sections suivantes, nous montrons comment nous avons transcrit quelques règles d'accessibilité en règles d'adaptation. Nous avons choisi des règles simples qui peuvent s'appliquer régulièrement et qui illustrent les adaptations à des problématiques persistantes (acuité visuelles) ou situationnelles (luminosité ambiante).

\subsubsection{Taille de police}

Nous n'avons trouvé aucune recommandation du W3C concernant la taille de police à appliquer par défaut. La taille par défaut utilisée semble être celle des navigateurs, généralement définie à $16 \mathrm{px}$, d'après le site W3C Online Web Tutorials (W3School).

Le WCAG recommande de donner la possibilité d'agrandir le texte jusqu'à $200 \%$ de sa taille d'origine (recommandation $n^{\circ} 1.4 .4$ ), et les EAPM du W3C indiquent, quant à elles, que certaines personnes ont besoin d'une taille de police plus grande pour distinguer les lettres $\left(n^{\circ}\right.$ 3.3.1). Toutefois, ils précisent aussi que d'autres personnes, ayant par exemple une bonne acuité visuelle, mais un problème de vision tunnélisée (altération de la vision périphérique), peuvent préférer de plus petites lettres pour visualiser davantage de mots. II n'est toutefois pas précisé à quelle taille de police correspond «plus petites lettres ». Nous avons donc choisi de traduire cela par la possibilité de réduire un texte jusqu'à $50 \%$ de sa taille d'origine. La taille de texte doit donc pouvoir être agrandie jusqu'à $200 \%$ de sa taille originale et réduite jusqu'à $50 \%$, ce qui, en se basant sur la taille par défaut de $16 \mathrm{px}$, se traduit par une fourchette allant d'un minimum de 8px à un maximum de 32px.

D'autres catégories de personnes peuvent bénéficier d'une adaptation de la taille de police. Ainsi, pour les personnes âgées, le projet WAI-AGE recommande l'utilisation de police de caractères d'une taille minimale de 12-14pt, soit 16-19px'1. (De Lara et al., 2010) indiquent dans le critère de réussite 2.4.14 que les liens adjacents doivent fournir suffisamment d'espace de clic pour éviter les erreurs -les utilisateurs âgés ont des difficultés à cliquer dans de petites zones de liens. (Dellaporta, 2007) recommande un texte de grande taille pour les liens hypertextes, une zone de prise en compte du clic suffisamment importante, et un espacement adéquat entre interacteurs afin d'éviter toute activation accidentelle.

Concernant les utilisateurs dyslexiques, (McCarthy et Swierenga, 2009) indiquent que l'un de principaux problèmes rencontrés est de trouver le texte trop petit. (De Santana et al., 2012) indiquent que la plus petite taille de texte recommandée se situe entre 12 et $14 \mathrm{px}$, et (Rello et al., 2013) recommandent une taille de 18pt, ce qui se traduit par 24px.

À partir de ces données, nous pouvons établir les règles élémentaires suivantes, avec les caractéristiques suivantes: visualAcuity décrit l'acuité visuelle de l'utilisateur, age son âge, dyslexic son niveau de dyslexie (plus ou moins vrai), tunnelVision sa perte de vision périphérique, fontSize la taille de police de caractères du texte, linkSize la taille des hyperliens.

(R1) if visualAcuity is low

$(\mathrm{R} 2)$ if age is senior

(R3) if dyslexia is high

(R4) if tunnelVision is high

(R5) if age is senior

\begin{abstract}
then fontSize is bigger
then fontSize is bigger

then fontSize is bigger

then fontSize is lower

then linkSize is bigger
\end{abstract}

\footnotetext{
1 Pour mémoire, le point est une ancienne unité d'imprimerie qui correspond à une taille en centimètres, et qui n'a donc pas de correspondance directe en informatique, mais qui est considéré comme valant 1,333 pixel.
} 
Les termes "bigger" et "lower" sont ici utilisés en prenant pour base la valeur de police de caractères par défaut du site. La première règle pouvant se traduire par "plus l'acuité visuelle est faible, plus il faut augmenter la taille de la police ". Ainsi, une taille de police par défaut définie à $16 \mathrm{px}$ sera agrandie ou diminuée en fonction des différentes caractéristiques. Par exemple à 20 px, si l'utilisateur est une personne âgée, ou à 22px si l'utilisateur est une personne âgée et qu'il a une acuité visuelle légèrement plus amoindrie.

\subsubsection{Niveau de contraste et gestion de couleurs}

La problématique des couleurs utilisées sur une IHM est complexe. En effet, comme l'ont dit Sarah Horton et Whitney Quesenbery, " sans contraste, le monde entier serait gris. Les concepteurs doivent respecter les besoins en contraste de couleur pour les personnes avec une vision ou une perception de couleurs dégradées. Les combinaisons de couleurs qui sont facilement distinguables pour quelqu'un avec une vision normale peuvent ne pas fonctionner pour des personnes souffrant de l'un des nombreux handicaps visuels existants. La perception des couleurs peut aussi être réduite par des facteurs environnementaux, comme les paramètres d'affichage ou les conditions lumineuses " (Horton et Quesenbery, 2014). Cet extrait illustre bien l'impératif de prendre en compte non seulement les différentes caractéristiques de l'utilisateur mais aussi d'autres paramètres comme sa plate-forme et son environnement pour arriver à un compromis acceptable. Dans ce contexte, la luminosité du dispositif est un élément important pouvant influer sur le contraste. Cependant, le réglage de cette luminosité depuis une application ou un site Internet n'est le plus souvent pas réalisable, voire jamais dans le cas des sites Internet. II n'est pas non plus évident que ce réglage automatique suffise à maintenir l'utilisabilité de l'IHM : nous avons tous l'expérience d'une IHM illisible en plein soleil malgré les tentatives de compensation de notre téléphone. L'IHM elle-même doit renforcer l'adaptation en modifiant la palette des couleurs utilisées.

Le WCAG recommande un contraste entre l'arrière-plan et le texte selon un ratio d'au moins 4.5:1 afin de respecter le niveau intermédiaire d'accessibilité (level AA recommandation 1.4.3), et un ratio d'au moins $7: 1$ pour le niveau élevé (level AAA recommandation 1.4.6).

La recommandation 2.4.2 des EAPM précise qu'un fond d'écran clair ne sera pas lisible, voire douloureux, pour des personnes atteintes d'une sensibilité accrue à la lumière, ou photophobie. Ces utilisateurs nécessiteront donc un fond d'écran plus sombre. De plus, la recommandation 3.1.2 du W3C traite du contraste entre le fond d'écran et le texte et précise que: 1) pour ces personnes photophobes, le texte a aussi besoin d'avoir une faible luminosité, et 2) d'autres personnes, incluant notamment les personnes âgées (qui subissent classiquement une perte de sensibilité à la lumière), nécessiteront un contraste élevé entre le fond d'écran et le texte, et que certains lisent notamment mieux avec un texte clair sur un fond sombre.

(De Santana et al., 2012) recommandent d'éviter les fonds d'écran d'un blanc pur pour les utilisateurs atteints de dyslexie car une part significative d'entre eux est trop sensible à sa luminosité (sensibilité scotopique) et verra le texte comme s'il vibrait ou s'il était flou. II est recommandé de privilégier des couleurs pastel, par exemple d'un texte bleu foncé sur un fond beige. En cas de dyslexie, il conviendra d'utiliser plutôt des fonds et des textes de couleurs pastel tout en maintenant un bon niveau de contraste.

Une autre catégorie de personnes pouvant avoir des problèmes de contraste sont les daltoniens qui représentent environ $8 \%$ de la population masculine et $2 \%$ de la population féminine (Laccarino et al., 2006). Le daltonisme se traduit par un "écrasement 》 des couleurs perçues qui induit un problème de contraste lorsque les couleurs perçues ont des luminosités trop proches les unes des autres. II conviendra d'éliminer les couleurs non perceptibles en fonction du type de daltonisme et de les remplacer par des couleurs de même luminosité que les couleurs initiales pour maintenir le contraste (Mochizuki et al., 2008). 
Enfin, en cas d'environnement sombre, (Rempel et al., 2011) recommandent d'utiliser une polarité négative (c'est-à-dire un texte clair sur un fond sombre) afin d'améliorer la lisibilité. Nous traduirons cette préconisation par la mise en place d'un arrière-plan de plus en plus sombre en fonction de la luminosité ambiante.

Nous pouvons déduire plusieurs règles de ces considérations, pour faire varier le niveau de contraste et/ou de luminosité, non pas en cherchant à calculer directement une couleur qui pourrait se trouver en contradiction avec la charte graphique de l'application, mais en évaluant dans quelles directions doit se faire l'adaptation puis en calculant les couleurs résultantes à partir de la charte graphique :

(R6) If photophobia is high

(R7) If age is senior

(R8) If dyslexia is high

(R9) If ambientLight is sunlight

(R10a) If ambientLight is low

(R10b) If ambientLight is veryLow

(R10c) If ambientLight is extraLow

(R11) If dyslexia is high

(R12) If ambientLight is sunlight

(R13) If photophobia is high

(R14) If Deuteranopia is high

(R15) If Protanopia is high

(R16) If Tritanopia is high then backgroundLuminosity is darker then backgroundLuminosity is lighter then backgroundLuminosity is darker then backgroundLuminosity is lighter then backgroundLuminosity is darker then backgroundLuminosity is darker then backgroundLuminosity is darker

then backgroundSaturation is pastel

then contrastLevel is higher then contrastLevel is lower then greenAvoidanceLevel is higher then redAvoidanceLevel is higher then blueAvoidanceLevel is higher

De même que pour les termes «bigger » et «lower » utilisés pour la taille de la police dans la sous-section précédente, les termes "darker", "lighter", « higher » et «lower » sont, là aussi, à corréler avec les valeurs par défaut utilisées sur le site pour les différentes caractéristiques de sortie.

Concernant la gestion des couleurs et le niveau de contraste, nous avons choisi de traduire les recommandations par l'utilisation de 3 variables: luminosité de l'arrière-plan (backgroundLuminosity), niveau de saturation de l'arrière-plan (backgroundSaturation) et niveau de contraste (contrastLevel). La luminosité et la saturation correspondent à deux des trois composantes du système de gestion des couleurs TSL (Teinte Saturation Luminosité). La troisième composante, la teinte, ne sera pas modifiée puisqu'elle correspond à la charte graphique du site web. Après avoir défini une nouvelle couleur d'arrière-plan en fonction de la luminosité et de la saturation, la variable de niveau de contraste est ensuite utilisée pour calculer une couleur de police de contraste suffisant avec la nouvelle couleur d'arrière-plan.

Enfin, les recommandations en termes de polarité ont été traduites ici par plusieurs règles pouvant se renforcer : à mesure que la luminosité baisse, la luminosité de l'arrièreplan est assombrie progressivement. 


\subsubsection{Espacements entre lettres, mots, lignes et paragraphes}

Concernant l'espacement entre les lettres, mots, lignes et paragraphes, la recommandation 1.4.8 du WCAG suggère de fournir à l'utilisateur le choix entre plusieurs espacements de lignes et de paragraphes avec, notamment, un choix d'espacement de ligne d'au moins 1.5 pour les blocs de texte, et un espacement de paragraphe d'au moins 1.5 fois l'espacement de lignes. La recommandation 1.4.12 du WCAG indique, quant à elle, que l'espacement entre les lettres doit être au moins 0.12 fois la taille de la police, l'espacement entre les mots, au moins 0.16 fois la taille de la police, l'espacement entre les lignes, au moins 1.5 fois la taille de la police, et l'espacement entre les paragraphes, au moins 2 fois la taille de la police.

Les EAPM indiquent que, pour pouvoir lire un texte, certaines personnes nécessitent plus d'espacement entre les lettres (recommandation 3.4.2), entre les mots (recommandation 3.4.3) et entre les lignes d'un bloc de texte (3.4.1) ;

(Dellaporta, 2007) recommande d'utiliser un espacement doublé entre les mots pour les personnes âgées. (Rello et al., 2012) recommandent, pour les personnes dyslexiques, un espacement de lettres augmenté de 7\%, un espacement de lignes 1.4 fois plus grand que l'espacement standard, et un espacement de paragraphe correspondant à 1 ligne de texte vide, soit 2 fois l'espacement de lignes.

De l'ensemble de ces recommandations, nous déduisons les règles suivantes où wordSpacing, lineSpacing et paragraphSpacing représentent l'espacement à utiliser entre, respectivement, les mots, les lignes et les paragraphes :

(R17) If visualAcuity is low

(R18) If dyslexia

(R19) If visualAcuity is low

(R20) If age is senior

(R21) If dyslexia is high

(R22) If visualAcuity is low

(R23) If age is senior

(R24) If dyslexia is high

(R25) If age is senior

(R26) If dyslexia is high

\begin{abstract}
then letterSpacing is bigger
then letterSpacing is bigger
\end{abstract}

then wordSpacing is bigger

then wordSpacing is bigger

then wordSpacing is bigger

then lineSpacing is bigger

then lineSpacing is bigger

then lineSpacing is bigger

then paragraphSpacing is bigger

then paragraphSpacing is bigger

\section{CAS D'ETUDE}

Maintenant que nous avons décrit notre architecture et détaillé le fonctionnement de la logique floue ainsi que les règles d'accessibilité que nous souhaitons utiliser, nous pouvons présenter comment notre architecture logicielle se concrétise sous forme d'un prototype.

\subsection{Le prototype}

L'architecture logicielle présentée en 5.1 a été mise en œuvre au moyen de technologies web et repose sur NodeJS, permettant d'exécuter du JavaScript aussi bien du côté serveur que du côté client. Les objets utilisés tels que les différents modèles de contexte ou la base de règles d'inférences, sont stockés dans des fichiers JSON. 
Concernant le moteur en logique floue, nous utilisons ExpressIF, un outil développé par CEA Tech appelé par un Web Service par une requête http.

Le moteur d'adaptation actuel s'appuie sur 81 règles en logique floue : 67 règles portant sur l'utilisateur, 6 règles pour la plate-forme et 8 règles pour l'environnement. II n'est pas spécifique à l'exemple de Bernard. Pour le cas de Bernard, les règles R6 à R13 n'ont pas été implémentées dans le prototype.

Notre prototype est un site web de réservation d'hôtel. II comprend 8 pages : (1) une page d'accueil de présentation de l'hôtel (Fig. 12) ; (2) une page détaillant les différentes chambres et suites proposées par l'hôtel (descriptions succinctes et détaillées) ; (3) une page pour le service de restauration et du bar ; (4) une page de présentation du service de petit-déjeuner ; (5) une page pour divers services (wifi, télévision, voiturier, ...) ; (6) une page de localisation, donnant l'adresse de l'hôtel, et les meilleurs moyens d'accès; (7) une page de réservation avec le formulaire de réservation et (8) une page de confirmation de réservation.

Fig. 12. Page d'accueil de l'hôtel (non adaptée - variant PC)

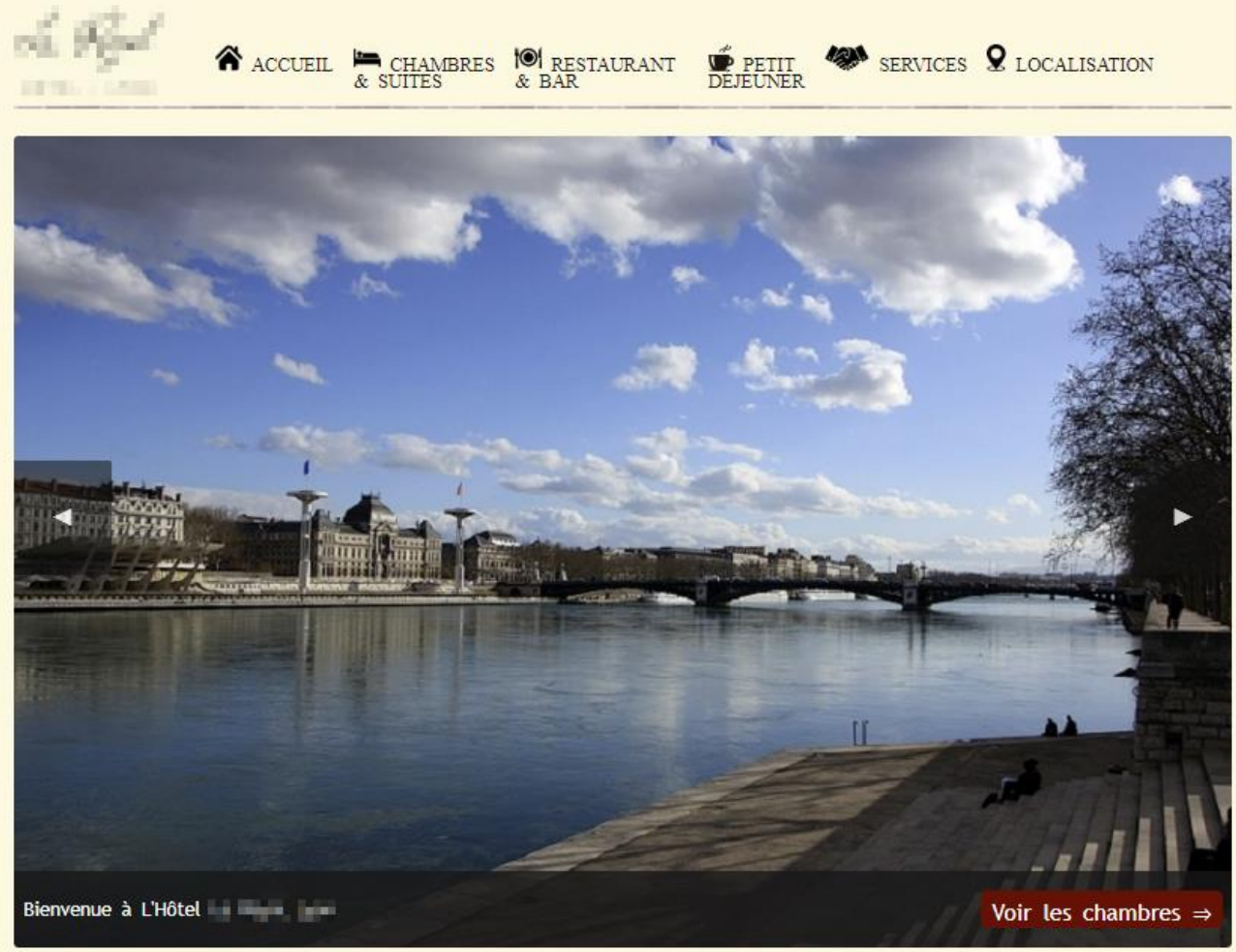

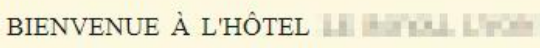

est une grande Maison de Famille de 72 chambres sur 5 étages dont 10 suites C'est un lieu où souffle l'esprit lyonnais, science de l'accueil mariant tradition et créativité, une adresse confidentielle pour voyageurs soucieux d'art de vivre, de discrete élégance et de service
naturellement généreux. Ce lieu offre un condensé de ce que Lyon, ses artisans et producteurs... proposent de mieux en Nous vous accueillons dans le confort, l'élégance et le charme avec un décor simple, moderne epuré. La couleur blanche associee au bleu ou au rouge des tissus donne une immédiat Les chambres ont été décorées avec des meubles contemporains signés Ligne Roset, côte à côte vec une bergere ancienne de Gilles Nouathac, les tissus signes Ralph Lauren, Pierre Fre Lelièvre, Design

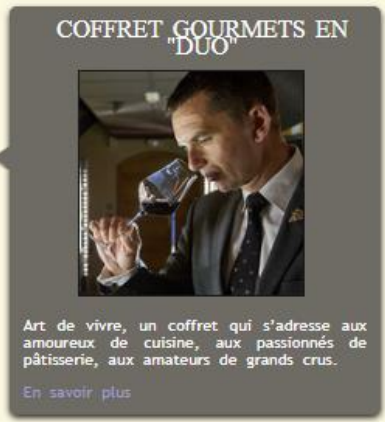


Les adaptations s'exécutent suffisamment rapidement pour ne pas gêner la navigation (environ $80 \mathrm{~ms}$ pour naviguer entre pages). Ce prototype sera testé à brève échéance lors d'une expérimentation avec plus d'une centaine d'utilisateurs finaux.

\subsection{La situation de Bernard}

Nous présentons ici l'exemple de Bernard en nous appuyant sur le prototype quand bien même certaines règles d'adaptation illustrées dans cet exemple ne sont pas implémentées, celles-ci ne présentant pas, a priori, de problème spécifique. Les adaptations présentées dans cette section sont donc celles envisagées.

La page de localisation du site de l'hôtel est présentée sur la figure 13. Sur la version non adaptée de cette IHM, la taille de police est définie à $12,8 \mathrm{px}$, ce qui est inférieur à la taille que nous avions identifiée comme taille par défaut (16px, voir section 5.1.1). L'arrièreplan est beige (en RGB : 255, 249, 222) tandis que le texte est gris foncé (en RGB : 24, 24, 24). Calculé avec les formules du WCAG, le ratio de contraste est de 16.8:1, ce qui est largement conforme aux préconisations (au minimum 7.1:1, voir section 6.1.2) pour une utilisation en luminosité normale.

Fig. 13. Page de localisation de l'hôtel (non adaptée - variant smartphone)

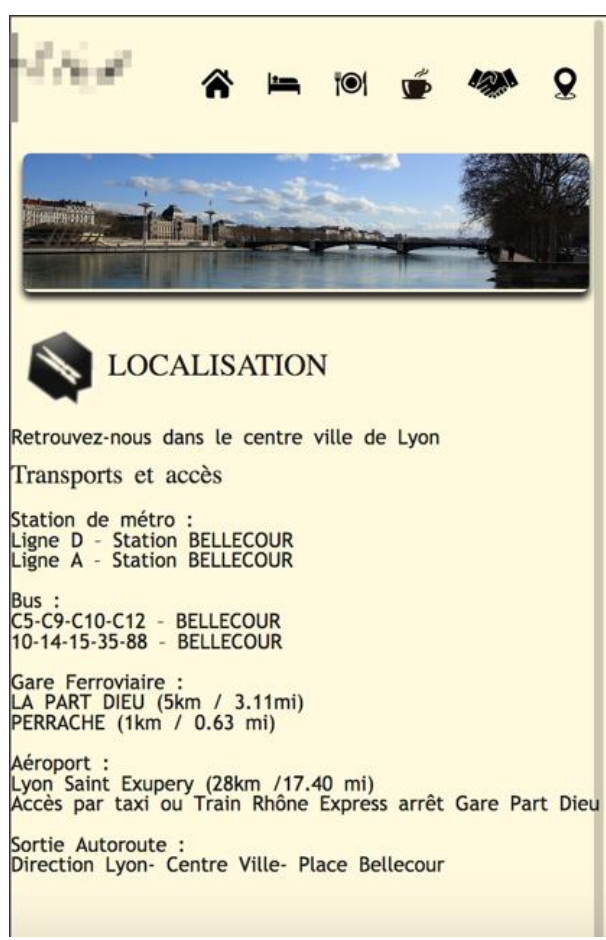

À l'ouverture du site web, le moteur d'inférence détecte la situation courante : il évalue la taille de l'écran de Bernard, déduit qu'il s'agit d'un smartphone et charge le variant correspondant. L'adaptation se base ensuite sur différentes caractéristiques du contexte d'usage, déclarées ou inférées. La luminosité ambiante est captée dans le but de détecter que Bernard, présentement dans le métro, est dans un environnement relativement sombre. Bernard a par ailleurs déclaré des caractéristiques personnelles: il a fourni sa date de naissance, il a indiqué souffrir de la cataracte (à un niveau qu'il a estimé « assez fort ») et de daltonisme dû à cette cataracte. II a aussi précisé ne pas être dyslexique, ni atteint de vision tunnélisée.

Si l'IHM ne s'adaptait pas à cette situation, Bernard n'aurait pas le choix : il devrait zoomer sur la page ou modifier le paramétrage de son navigateur pour agrandir la taille de la police de caractères. Cependant, n'ayant qu'une main libre du fait de sa canne, zoomer à l'aide d'un pincement serait complexe. De plus, outre le fait de nécessiter une action 
utilisateur, le zoom proportionnel entraîne généralement le besoin de scroller horizontalement pour chaque ligne à lire, ce qui n'est pas recommandé (WCAG), encore moins pour les personnes âgées (WAI-AGE). II ne pourrait pas facilement augmenter l'espacement entre les lettres, mots et paragraphes, alors que, nous l'avons vu, c'est un facteur de confort.

Dans le cas présent, avec une interface adaptative, le moteur activerait les règles floues impactées par l'acuité visuelle de Bernard, son âge, sa photophobie, ainsi que la luminosité ambiante : R1, R2, R5, R6, R7, R10, R13, R17, R19, R20, R22, R23 et R25. Ces règles nous renverraient une valeur pour la taille de police de tout texte (R1 et $R 2$ ) et la taille de police des hyperliens en particulier (R1, R2 et R5), le niveau de luminosité et de saturation de l'arrière-plan ( $R 6, R 7$ et $R 10)$, le niveau de contraste entre arrière-plan et texte (R13), et les divers espacements (R17, R19, R20, R22, R23 et R25). Un point important ici est que les règles peuvent se renforcer mutuellement ou s'opposer. Concernant l'espacement entre les mots, le fait que Bernard soit considéré comme âgé entraînerait une augmentation de l'espacement (R20) tout comme le fait qu'il soit considéré comme ayant une acuité visuelle dégradée, entraînant le même effet (R19). En termes de niveau de contraste et de gestion des couleurs, nous avons ici des règles de finalités opposées: d'une part, un assombrissement de l'arrière-plan dû à la photophobie (R6) et à la diminution de la luminosité ambiante (R10), et d'autre part, un éclaircissement en raison de l'âge de Bernard (R7). Ici, la combinaison du lot de règles $R 10$ ainsi que la règle $R 6$ auraient plus de poids que la règle $\mathrm{R} 7$, entraînant la mise en place d'une interface assombrie (figure 14a).

Fig. 14. Page de localisation de l'hôtel adaptée à Bernard pour smartphone et pour un environnement sombre (a) et un environnement lumineux (b)
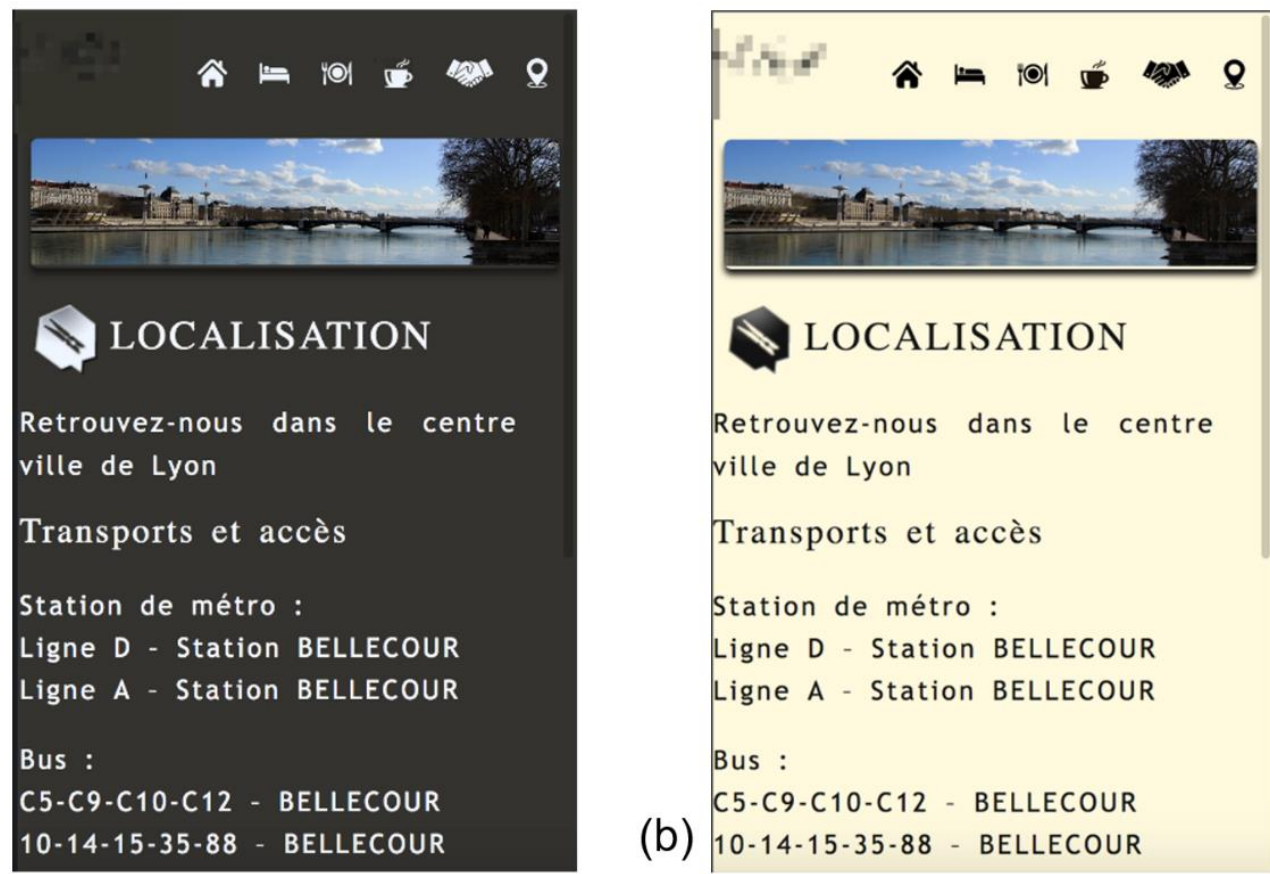

Une fois arrivé à sa station de métro, Bernard reprend son téléphone afin de regarder comment atteindre l'hôtel. Bernard sort de la station de métro tout en consultant la page de localisation de l'hôtel : la luminosité ambiante augmente progressivement jusqu'à atteindre le plein soleil à l'extérieur. Dès lors, le système réagirait en activant de moins en moins le lot de règles $\mathrm{R} 10$ et en lançant l'activation de la règle $\mathrm{R} 12$. La diminution de $\mathrm{R} 10$ entraînerait un retour à la normal en terme de luminosité d'arrière-plan, et l'IHM reviendrait à sa version standard avec son fond beige clair. Cependant, la règle R12 déclencherait une augmentation du niveau de contraste avec le texte. La condition « if sunlight » étant de plus en plus vérifiée au fil de l'avancement de Bernard vers la sortie, la règle préconiserait un ratio de contraste de plus en plus fort, ce qui entrerait en contradiction avec les conclusions de la règle $\mathrm{R} 13$ : la 
cataracte induisant une légère photophobie, la condition « if photophobe » a une probabilité non nulle et tend donc à légèrement atténuer le contraste. La combinaison des règles en logique floue permet de trouver un compromis sans avoir besoin de déterminer un système de priorisation : le contraste préconisé dans une telle situation serait légèrement inférieur à celui que recommanderait la règle sur la luminosité seule (figure 14b).

\section{DISCUSSION}

Nous avons illustré avec des exemples concrets comment notre prototype prend en compte les règles d'accessibilité et de là, améliorer la prise en compte de l'accessibilité. Ce prototype est de taille réaliste pour un site web. Les règles à mettre à œuvre simultanément sont suffisamment nombreuses (81) pour obtenir une combinaison de règles réaliste.

Avec l'exemple de Bernard, nous avons détaillé un ensemble de 26 règles. Nous avons choisi une situation qui nécessitait l'activation simultanée de 13 d'entre elles. Enfin, avec l'exemple du calcul du niveau de contraste, nous avons montré comment les règles peuvent s'opposer ou se renforcer, et comment le moteur d'adaptation en logique floue, grâce à la défuzzification, produirait le résultat idoine.

Dans (Giuffrida et al., 2019), une évaluation avec des experts a permis de tester la pertinence des choix du moteur en logique floue. II est maintenant nécessaire de tester la combinaison des règles avec des utilisateurs finaux. Pour ce faire, une expérimentation est en cours de réalisation avec le prototype présenté dans cet article afin de voir s'il permet de répondre convenablement à nos problématiques d'accessibilité.

La plupart des recommandations que nous avons trouvées dans la littérature est transcriptible en règles d'adaptation. Pour cela, il est nécessaire d'avoir une métrique qui caractérise les éléments de contexte, les seuils, et les actions pour ces seuils. Par exemple, pour la recommandation du WAI-AGE sur l'utilisation de police de caractères d'une taille minimale de 12-14pt pour les personnes âgées, nous avons une métrique (l'âge), un seuil (“personne âgée », traduit ici par un âge supérieur à 55 ans), et une action (taille de police minimale). Toutefois, métrique, seuil et action peuvent être difficiles à identifier. Tel est le cas, par exemple, de la règle 1.1.1 du WCAG qui spécifie que "si le contenu non textuel vise d'abord à créer une expérience sensorielle spécifique, l'équivalent textuel fournit au moins une identification descriptive de ce contenu non textuel ». A moins d'annoter le contenu des IHM avec des indicateurs d'intention, il n'est pas possible de détecter automatiquement que l'intention du concepteur est de créer une expérience sensorielle. Il est donc impossible d'exprimer que l'identification descriptive doit être proposée par défaut si l'utilisateur n'est pas à même de partager cette expérience sensorielle.

En outre, la littérature fournit peu de règles à respecter en cas de contexte d'usage complexe et dynamique, par exemple pour une personne ayant une combinaison de plusieurs handicaps (permanents ou situationnels). Tel est le cas de Bernard, pourtant un exemple simplifié qui ne tient compte que d'un nombre restreint de caractéristiques. Cette question difficile a été abordée dans quelques travaux comme la thèse d'Amélie Roche en 2015. Toutefois, à notre connaissance, ce problème a été traité davantage sous l'angle des techniques de conception d'IHM pour les polyhandicapés (et notamment le recueil de besoins) que sous l'angle de la définition de systèmes adaptatifs.

Dans un système adaptatif, l'une des difficultés est que deux handicaps non exclusifs peuvent avoir des recommandations qui se renforcent ou s'opposent. On pourrait par exemple souhaiter augmenter la taille de police (car un utilisateur a une mauvaise acuité visuelle) mais aussi la réduire (car cet utilisateur est atteint de vision tunnélisée). Une adaptation ne prenant en compte que l'un des handicaps pourrait poser problème: l'utilisateur pourrait se retrouver pénalisé en raison du second handicap qui nécessiterait une adaptation opposée. Notre outil permet ici de calculer un compromis entre les deux recommandations, via la logique floue et sa gestion de la combinatoire des règles. 
A l'inverse, avant de généraliser l'approche, il conviendra de répondre à trois questions : (a) toutes les règles d'adaptation peuvent-elles se ramener à des séries de règles floues simples ? (b) la recherche de compromis ne risque-t-elle pas d'être contre-productive dans certains cas ? et (c) les utilisateurs sont-ils davantage satisfaits avec un système adaptatif qu'avec un système non adaptable ? Ce dernier point est celui que nous allons aborder expérimentalement à court terme.

\section{CONCLUSION}

Avec la diffusion massive de l'utilisation d'Internet, l'accessibilité des interfaces est un besoin toujours plus prégnant. Cependant, même si de nombreux travaux ont abordé cette thématique, il demeure des verrous tels que l'incertitude du contexte d'usage et de la combinaison de multiples règles d'adaptation. Dans cet article, nous avons montré comment Fuzzy4U permet d'améliorer dynamiquement l'accessibilité des IHM grâce à un moteur d'adaptation utilisant la logique floue.

Nous avons aussi précisé comment des recommandations d'accessibilité statiques, sont traduisibles en règles d'adaptation floues, et comment ces règles peuvent ensuite être activées dynamiquement en fonction de la situation de l'utilisateur pour améliorer l'accessibilité des IHM. Pour cela, la logique floue dispose d'une gestion intrinsèque de l'incertitude et de la combinatoire des règles, permettant de les combiner pour se renforcer ou se modérer mutuellement afin de parvenir à un résultat moyenné. Nous avons montré sur un prototype comme elle pouvait être mises en œuvre. Ce prototype doit maintenant être testé auprès d'utilisateurs représentatifs pour évaluer la pertinence des adaptations.

Au vu des difficultés à identifier les recommandations d'accessibilité, dispersées dans la littérature, et à les formaliser en règles codées dans le cas de contextes d'usage complexes, il serait intéressant de créer un référentiel de règles basées sur les recommandations.

\section{LES PERSPECTIVES D'APPLICATION DE CETTE RECHERCHE :}

$\checkmark$ L'amélioration de l'accessibilité des IHM au travers d'un moteur d'adaptation en logique floue

$\checkmark$ La prise en charge des handicaps multiples grâce à la combinatoire des règles

$\checkmark$ La création d'un référentiel de règles d'accessibilité

\section{REFERENCES}

- Abrahão, S., Bordeleau, F., Cheng, B., Kokaly, S., Paige, R. F., Störrle H. et WhITTLE, J., 2017. User Experience for Model-Driven Engineering: Challenges and Future Directions. In 2017 ACM/IEEE 20th International Conference on Model Driven Engineering Languages and Systems (MODELS).

- Alm, N., Dye, R., Gowans, G., Campbell, J., Astell, A. J. et Ellis, M., 2007. A COMMUNICATION SUPPORT SYSTEM FOR OldER PEOPLE WITH DEMENTIA. IEEE COMPUTER SOCIETY, WASHINGTON.

- AkIKI, P., Bandara A. K., Yu Y., 2014. Adaptive Model-Driven User INTERface DeVelopment Systems. ACM Computer SuRVey, 47 (1), ARticle 9 (MAi 2014), 33 PAGES.

- AKIKI, P., 2016. EngineERING AdAPtive Model-Driven USER INTERFAces fOR ENTERPRISE APPLICATIONS. IN IEEE TRANSACTIONS ON SOFTWARE ENGINEERING, 42 (12), PP. 1118-1147.

- Beka Be nguema M., Kolski C., Malvache N., Waroux D., 2000. Design of human ERROR TOLERANT INTERFACE USING FUZZY LOGIC. ENGINEERING APPLICATIONS OF ARTIFICIAL INTELLIGENCE, 13 (3), PP. 279-292.

- Berner, J., 2014. Psychosocial, Socio-Demographic and Health Determinants in 
INFORMATION COMMUNICATION TECHNOLOGY USE BY OLDER-ADULTS. THĖSE DE DOCTORAT, BLEKINGE INSTITUTE OF TECHNOLOGY, SUĖDE.

- Blouin, A., Morin, B., Beaudoux, O., Nain, G., Albers, P. et Jezequel J.-M., 2011. COMBINING ASPECT-ORIENTEd MOdELING WITH PROPERTY-BASEd REASONING TO IMPROVE USER INTERFACE ADAPTATION. IN PROCEEDINGS OF THE 3RD ACM SIGCHI SYMPOSIUM ON ENGINEERING INTERACTIVE COMPUTING SYSTEMS, PISE, ITALY, PP. 85-94.

- Blumendorf, M., Feuerstack S. et Albayrak, S., 2008. Multimodal User InTerfaces

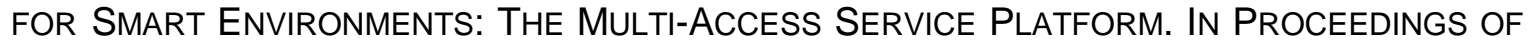
THE WORKING CONFERENCE ON ADVANCED VISUAL INTERFACES, NAPOLI, 2008, PP. 478-479.

- Bouchon-Meunier B., 2007. La logique floue. Presses Universitaires de France PUF (ED.), 2007.

- Calvary, G., Coutaz, J., Thevenin, D., Limbourg, Q., Boulllon, L. et Vanderdonckt, J., 2003. A UNIFYING REFERENCE FRAMEWORK FOR MULTI-TARGET USER INTERFACES. INTERACTING WITH COMPUTERS, 15 (3), PP. 289-308.

- CaO, J., Xing, N., Chan, A., Feng, Y. et Jin, B., 2005. Service adaptation using fuzzy THEORY IN CONTEXT-AWARE MOBILE COMPUTING MIDDLEWARE, IN PROCEEDINGS OF THE 11TH IEEE INTERNATIONAL CONFERENCE ON EMBEDDED AND REAL-TIME COMPUTING SYSTEMS AND APPLICATIONS (RTCSA'05), HONG KONG, CHINA, PP. 496-501.

- Ceret, E., Dupuy-Chessa, S., Calvary, G. et Bittar, M., 2016. System and method FOR MAGNETIC ADAPTATION OF A USER INTERFACE, FRANCE INPI BREVET TPI2015053, 07 072015 ET 07072016.

- Chesta C., Paterno F. et Santoro C., 2004. Methods and ToOls for Designing and DeVeloping Usable multi-Platform interactive applications, PsychNology JOURNAL, VOL. 2, PP. 123-139.

- Coutaz J., 2006. Meta-User Interfaces for Ambient Spaces. In Proceedings of the 5TH INTERNATIONAL CONFERENCE ON TASK MODELS AND DIAGRAMS FOR USERS INTERFACE DESIGN (TAMODIA'06), Hasselt, Belgium, pp. 1-15.

- COUTAZ J., 2010. User Interface Plasticity: Model Driven Engineering to the Limit!. ACM, ENGINEERING INTERACTIVE COMPUTING SYSTEMS (EICS 2010) INTERNATIONAL CONFERENCE, KeYNOTE PAPER, BERLIN, GERMANY, PP. 1-8.

- Cueva-Fernandez, G., Espada, J. P., Garcia-Diaz, V., Crespo, R. G. et GarciaFERNANDEZ, N., 2016. FuZZY SYSTEM TO ADAPT WEB VoICE INTERFACES DYNAMICALLY IN A Vehicle Sensor tracking Application Definition, in Soft Computing, 20 (8), PP. 3321-3334.

- De Lara, S., Watanabe, W., dos Santos, E. et Fortes, R., 2010. Improving WCAG for Elderly Web ACCESSIBILITY, IN PROCEedings OF THE 28TH ACM INTERNATIONAL CONFERENCE ON DESIGN OF COMMUNICATION (SIGDOC'10), SÃo PAULO, BRÉSIL.

- De Santana, V. F., De Oliveira, R., Almeida, L. D. A. et Baranauskas, M. C. C., 2012. Web Accessibility and PEOPLE WITH DYSLEXIA, IN PROCEEDINGS OF the INTERNATIONAL CROSS-DISCIPLINARY CONFERENCE ON WEB ACCESSIBILITY (W4A'12).

- Dellaporta, A., 2007. Web Accessibility and the Needs Of USERS With Disabilities, IN Advances in Universal Web Design and Evaluation: Research, Trends and OPPORTUNITIES. IGI GLOBAL, 2007, PP. 1-24.

- Desruelle, H., Blomme, D. et Gielen, F., 2011. Adaptive Mobile Web Applications: A Quantitative EVAluation APPROACH, IN INTERnational CONFERENCE ON WeB ENGINEERING, SPRINGER BERLIN HEIDELBERG, PP. 375-378.

- Deuff, D., Cosquer, M., 2013. User-Centered Agile Method. FOCUS Series, WileyISTE ED., FIRST EDITION, 127 PAGES, ISBN 978-1848214538. 
- Dey, A.K., 2001. Understanding and Using Context, in Personal UbiQuitous COMPUTING, 5 (1), PP. 4-7.

- Edmonds, E.A., 1981. Adaptive Man-Computer Interfaces. In CoOmbS, M. J. AND Alty, J. L., (EDS.), COMPUTING SKILLS AND THE USER INTERFACES. ACADEMIC PRESS, LONDON.

- Florins, M., 2006. Graceful Degradation: a Method for Designing Multiplatform GRAPHICAL USER INTERFACES. THĖSE DE DOCTORAT, LOUVAIN-LA-NEUVE, BELGIQUE.

- Gajos, K. Z., Weld, D. S., et Wobbrock J. O., 2010. Automatically Generating Personalized User INTERFACES With SupPle. ARTIFICIAL INTELLIGENCE, 174 (12-13), AUGUST 2010, PP. 910-950.

- Genaro Motti, V., Mezhoudi, N. et Vanderdonckt, J. (Eds.), 2012. Machine Learning IN THE SUPPORT OF CONTEXT-AWARE ADAPTATION. WORKSHOP ON CONTEXT-AWARE ADAPTATION OF SERVICE FRONT-ENDS (PISA, 13/11/2012). IN: FRANCISCO JAVIER

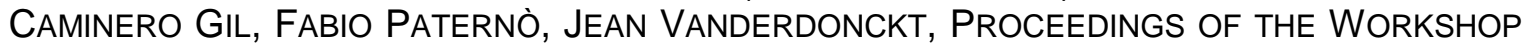
ON CONTEXT-AWARE ADAPTATION OF SERVICE FRONT-ENDS, 2012.

- Ghiani, G., Manca, M., Paterno, F. et Santoro, C., 2017. Personalization of CONTEXT-Dependent Applications Through Trigger-Action Rules. ACM TRANSACTIONS ON COMPUTER-HUMAN INTERACTION (TOCHI), 24(2), ARTICLE 14, 33 PAGES.

- Giuffrida, T., Dupuy-Chessa, S., Poli, J.-Ph., Ceret, E., 2019. Fuzzy4U: A Fuzzy Logic SYSTEM FOR USER INTERFACES ADAPTATION. IN IEEE THIRTEEN INTERNATIONAL CONFERENCE ON RESEARCH CHALlENGES IN INFORMATION SCIENCE (RCIS), MAY 29-31, 2019, BRUSSELS, BELGIUM.

- Haut conseil de la famille, de l'enfance et de l'age (HCFEA), 2019. Avis du Conseil DE L'AGE SUR LA DEMARCHE MONALISA ET SUR LA POLITIQUE DE MAINTIEN DU LIEN SOCIAL ET DE LUTTE CONTRE L'ISOLEMENT DES PERSONNES AGEES, ADOPTE LE 16 MAI 2019, HTTP://WWW.HCFEA.FR.

- Horton, S. et Quesenbery, W., 2014. A Web for Everyone: Designing Accessible USER EXPERIENCES, ROSENFELD MEDIA ED., 288 PAGES.

- Iaccarino, G., Malandrino, D., Del Percio, M. et Scarano, V., 2006. Efficient EdgeSERVICES FOR COLORBLIND USERS. PROCEEDINGS OF THE 15TH INTERNATIONAL CONFERENCE ON WORLD WIDE WEB (WWW'06), EDINBURGH, SCOTLAND.

- ISO 9241-210:2010. ERgONOMICS Of HUMAN-SYSTEM INTERACTION - PART 210: HUMANCENTRED DESIGN FOR INTERACTIVE SYSTEMS. 2010. ISO, GENEVA, SWITZERLAND.

- Jasberg, K. et Sizov, S., 2018. Neuroscientific User Models: The Source of UNCERTAIN USER FEEDBACK AND POTENTIALS FOR IMPROVING WEB PERSONALISATION. IN: Hacid H., Cellary W., Wang H., Paik HY., Zhou R. (EDS.) Web InFORMation SYSTEMS ENGINEERING (WISE 2018). WISE 2018. LECTURE NOTES IN COMPUTER SCIENCE, VOL 11234. SPRINGER, CHAM

- Jung, S. G., An, J., Kwak, H., Salminen, J. et Jansen, B. J., 2018. Assessing the

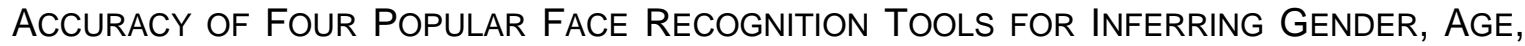
AND RACE. IN TWELFTH INTERNATIONAL AAAI CONFERENCE ON WEB AND SOCIAL MEDIA.

- Kolekar, S., Pai, R. et Manohara, M. M., 2018. Rule-based Adaptive User Interface FOR ADAPTIVE E-LEARNING SYSTEM. IN EDUCATION AND INFORMATION TECHNOLOGIES 24, PP. 613-641.

- Kolski C. et le Strugeon, E., 1998. A Review of Intelligent human-Machine INTERFACES IN THE LIGHT OF THE ARCH MODEL. INTERNATIONAL JOURNAL OF HUMANCOMPUTER INTERACTION, 10 (3), PP. 193-231.

- Kurniawan, S. H., King, A., Evans, D. G. et Blenkhorn, P. L., 2006. Personalising Web Page Presentation for Older People. Interacting With COMPuters, 18(3), PP. 
457-477.

- Law, E. L.-C., Roto V., Hassenzahl, M., Vermeeren, A. P.O.S. et Kort, J., 2009. UNDERSTANDING, SCOPING AND DEFINING USER EXPERIENCE: A SURVEY APPROACH. IN PROCEEDINGS OF THE SIGCHI CONFERENCE ON HUMAN FACTORS IN COMPUTING SYSTEMS (CHI '09). ACM, NEW YORK, NY, USA, PP. 719-728.

- Lehmann, G., Rieger, A., Blumendorf M. et Albayrak, S., 2010. A 3-layer ARCHITECTURE FOR SMART ENVIRONMENT MODELS. IN 8TH IEEE INTERNATIONAL

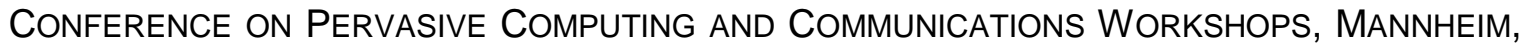
PP. 636-641.

- Loranger, H., 2017. Plain language Is for Everyone, Even Experts. Nielsen NORMAN GROUP. ACCESSIBLE EN LIGNE : HTTPS://WWW.NNGROUP.COM/ARTICLES/PLAINLANGUAGE-EXPERTS/.

- LÜtJen, M., Kreowski, H.-J., Franke M., Thoben, K.-D. et Freitag, M., 2014. ModelDRIVEN LOGISTICS ENGINEERING - CHALLENGES OF MODEL AND OBJECT TRANSFORMATION. PROCEDIA TECHNOLOGY, VOL. 15, PP. 303-312. ISSN 2212-0173.

- Marçal de Oliveira, K., Girard, P., Guidini Gonçalves, T., Lepreux, S., and Kolski, C., 2015. TEACHING TASK ANALYSIS FOR USER INTERFACE DESIGN: LESSONS LEARNED FROM

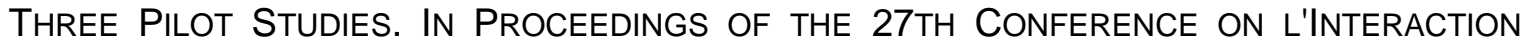
HOMME-MACHINE (IHM '15). ACM, NeW YORK, NY, USA, ARTICLE 31, 6 PAGES.

- McCarthy, J. E. et Swierenga, S. J., 2009. What We Know about Dyslexia and Web ACCESSIBILITY: A RESEARCH REVIEW. UNIVERSAL ACCESS IN THE INFORMATION SOCIETY, 9(2), PP.147-152.

- Meixner, G., Paterno, f. et Vanderdonckt, J., 2011. Past, Present, and Future of Model-BASEd USER INTERFACE DeVELOPMENT. I-COM 10, 3 (NOVEMBER 2011), 2-10.

- Miñon, R., Paterno, F., Arrue, M. et Abascal, J., 2016. Integrating Adaptation Rules for PeOple With Special NeEds in Model-based UI DeVelopment Process. UNIVERSAL ACCESS IN THE INFORMATION SOCIETY, 15 (1), PP. 153-168.

- Mochizuki, R., Nakamura, T., Chao, J. et lenz, R., 2008. Color-Weak Correction by DISCRIMINATION THRESHOLD MATCHING, IN CONFERENCE ON COLOUR IN GRAPHICS, IMAGING, AND VISION, VOL. 2008, N 1, PP. 208-213, SOCIETY FOR IMAGING SCIENCE AND TECHNOLOGY.

- Mussbacher, G., Amyot, D., Breu, R., Bruel, J.-M., Cheng, B.H.C., Collet, P., Combemale B., France, R.B., Heldal, R., Hill, J., Kienzle, J., Schöttle, M., SteimanN, F., Stikkolorum, D. et Whittle, J., 2014. The Relevance of Model-Driven ENGINEERING THIRTY YEARS FROM NOW. IN MODEL-DRIVEN ENGINEERING LANGUAGES AND SYSTEMS (MODELS'14), LECTURE NOTES IN COMPUTER SCIENCE, VOL. 8767, PP. 183-200.

- Myers, B., Hudson, S. E., and Pausch, R., 2000. Past, Present, and Future of User INTERFACE SOFTWARE TOOLS. ACM TRANSACTION ON COMPUTER-HUMAN. INTERACTION (TOCHI), 7 (1), (MARCH 2000), PP. 3-28.

- Nguyen, T.-D., Vanderdonckt, J. et Seffah, A., 2016. UIPLML: A Pattern ToOl for ENGINEERING MULTI-PLATFORMS INFORMATION SYSTEMS. IN TENTH INTERNATIONAL CONFERENCE on RESEARCH Challenges in INFORMATION SCIENCE (RCIS), JUNE 1-3, 2016, GRENOBLE, FRANCE.

- Nyongesa, H. O., Shicheng, T., Maleki-Dizaji, S., Huang, S. T. et SiddiQI, J., 2003. adaptive Web Interface Design Using Fuzzy Logic, in Proceedings IEEE/WIC INTERNATIONAL CONFERENCE ON WEB INTELLIGENCE (WI 2003), PP. 671-674.

- Papatheocharous, E., Belk, M., Germanakos, P. et Samaras, G., 2012. Proposing a

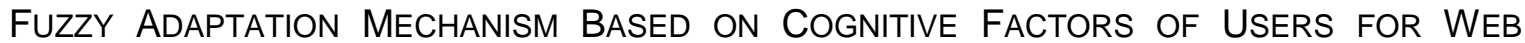


PERSONALIZATION. IN 8TH INTERNATIONAL CONFERENCE ON ARTIFICIAL INTELLIGENCE ApPlications AND INNOVATIONS (AIAI), SEPT. 2012, HALKIDIKI, GREECE, PP.135-144.

- Paterno, F., Santoro, C., et Spano, L. D., 2009. Maria: A Universal, Declarative, MULTIPLE ABSTRACTION-LEVEL LANGUAGE FOR SERVICE-ORIENTED APPLICATIONS IN UBIQUITOUS ENVIRONMENTS. ACM TRANSACTIONS ON COMPUTER-HUMAN INTERACTION (TOCHI), 16 (40, 2009.

- Peissner, M., Häbe, D., Janssen, D. et Sellner, T., 2012. MyUl: Generating ACCESSIBLE USER INTERFACES FROM MULTIMOdAL DESIGN PATTERNS. IN PROCEEDINGS OF THE 4TH ACM SIGCHI SYMPOSIUM ON ENGINEERING INTERACTIVE COMPUTING SYSTEMS, NEW YORK.

- Quillion-Dupré, L., Monfort, E. et Rialle, V., 2018. Tablette tactile et adultes ÁGÉS, APPORTS D'UN PROGRAMME D'INITIATION ADAPTÉ AUX TROUBLES NEUROCOGNITIFS, EDUCATION \& FORMATION - E-311, NOVEMBRE 2018, PP. 43-59.

- Rello, L., Kanvinde, G., et Baeza-Yates, R., 2012. Layout Guidelines for Web Text

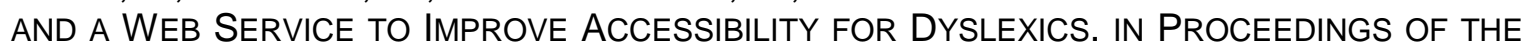
INTERNATIONAL CROSS-DISCIPLINARY CONFERENCE ON WEB ACCESSIBILITY (W4A'12).

- Rello, L., Pielot, M. et Marcord, M.-C., 2013. Size Matters (Spacing Not): 18 points FOR A DYSLEXIC-FRIENDLY WIKIPEDIA. IN PROCEEDINGS OF THE 10TH ACM INTERNATIONAL CROSS-DISCIPLINARY CONFERENCE ON WEB ACCESSIBILITY (W4A'13), RIO DE JANEIRO, BRAZIL, PP.1-4.

- Rempel, A. G., Mantiuk, R. et Heidrich, W., 2011. Display Considerations for IMPROVEd Night VISION PERFORMANCE. IN PROCEEDINGS OF THE 19TH COLOR AND IMAGING CONFERENCE, PP. 191-194(4), SOCIETY FOR IMAGING SCIENCE AND TECHNOLOGY PUB.

- Richards, J. T. et hanson, V. L., 2004. Web acCessibility: a broader VieW. IN PROCEEDINGS OF THE 13TH ACM INTERNATIONAL CONFERENCE ON WORLD WIDE WEB, PP. 72-79.

- Riding, R. et Cheema, I. 1991. Cognitive Styles - an OVerview And INTEGRation. IN EDUCATIONAL PSYCHOLOGY, VOL. 11, PP. 193-215.

- Schmutz, S., Sonderegger, A. et Sauer, J., 2017. Effects of Accessible Website Design ON NONDISABled USERS: Age and DeVICE as Moderating Factors. IN ERGONOMICS 61, PP. 1-39.

- Schneider-Hufschmidt, M., KÜHME, T., et Malinkowski, U. (EdS.) (1993). AdAPtive USER INTERFACES: PRINCIPLES AND PRACTICE. NORTH HOLLAND.

- Sottet, J.S., Ganneau, V., Calvary, G., Coutaz, J., Demeure, A., Favre, J.-M., Demumieux, R., 2007. Model-Driven AdAPtation fOR Plastic USER INTERFACES. IN: Baranauskas C., Palanque P., Abascal J., Barbosa S.D.J. (EdS.) Human-Computer INTERACTION - INTERACT 2007. LECTURE NOTES IN COMPUTER SCIENCE, VOL. 4662. SPRINGER, BERLIN, HEIDELBERG.

- Soul, M., Abed, M. et Ghedira, K., 2013. FuzZy Logic Approach for Adaptive Systems DESIGN. IN: KUROSU M. (EDS.) HUMAN-COMPUTER INTERACTION. TOWARDS INTELLIGENT AND IMPLICIT INTERACTION. HCI 2013. LECTURE NOTES IN COMPUTER SCIENCE, VOL. 8008. SPRINGER, BERLIN, HEIDELBERG, PP. 141-150.

- Thevenin, D. et Coutaz, J., 1999. Plasticity of User InTERfaces: Framework and Research Agenda. PROCEEDING OF IFIP CONFERENCE ON HUMAN COMPUTER INTERACTION INTERACT. AUGUST 30 - SEPTEMBER 3,1999. EDINBURGH, SCOTLAND. SASSE, A. AND JOHNSON, C. (EDS.), IFIPIOS PRESS.

- Thevenin, D., Coutaz, J. et Calvary, G., 2003. A Reference Framework for the DEVElopMent of Plastic USER INTERFACES, IN MULTI-DEVICE AND MULTI-CONTEXT USER 
INTERFACES: ENGINEERING AND APPLICATIONS FRAMEWORKS.

- Virtual User Modeling and Simulation Cluster (VUMS), 2013. AcCessible en ligne : HTTP://VUMS.ITI.GR/INDEXB60B.HTML?PAGE_ID=44.

- Whittle, J., Hutchinson, J., Rouncefield, M., 2014. The State of Practice in ModelDRIVEN ENGINEERING, IEEE SOFTWARE, 31(3), PP. 79-85.

- Wobbrock, J., 2019. Situationally aware Mobile DeVices for OVercoming Situational IMPAIRMENTS. IN PROCEEDINGS OF THE ACM SIGCHI SYMPOSIUM ON ENGINEERING INTERACTIVE COMPUTING SYSTEMS (EICS '19). ACM, NEW YORK, NY, USA, PP. 1-18.

- Yigitbas, E., Sauer, S. et Engels, G., 2017. Adapt-UI: an IDE Supporting ModelDriven DeVElopment of Self-AdaPtive UIS. IN PROCEedings of the ACM SIGCHI SYMPOSIUM ON ENGINEERING INTERACTIVE COMPUTING SYSTEMS, LISBON, 2017.

- Web AcCessibility Initiative (WAI), 2015. IntRoduction to Web AcCessibility. ACCESSIBLE EN LIGNE : HTTPS://WWW.W3.ORG/WAI/FUNDAMENTALS/ACCESSIBILITY-INTRO.

- Yang, S.J. et ShaO, N.W., 2007. Enhancing Pervasive Web AccessibiLity With RuleBased AdAPtation StRategy. EXPERT Systems With ApPlications, 32 (4), PP. 11541167.

- ZAdeh, L., 1965. Fuzzy SETS, IN INFORMATION AND CONTROL, 8 (3), PP. 338-353.

- Zadeh, L., 1975. The Concept of a Linguistic Variable and its Application to APPROXIMATE, IN INFORMATION SCIENCES, VOL. 9, PP. 43-80. 


\section{BIOGRAPHIE}

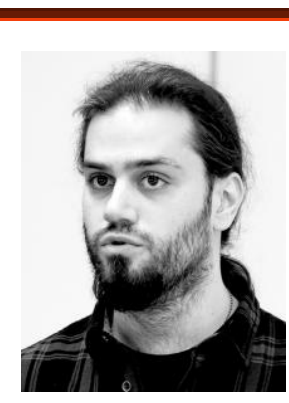

\section{Tanguy Giuffrida}

est doctorant en informatique et marketing, au sein de l'équipe Ingénierie de l'Interaction HommeMachine du Laboratoire d'Informatique de Grenoble. Ses travaux de recherche sont centrés sur l'adaptation des IHM dans un but d'amélioration de l'accessibilité des utilisateurs.

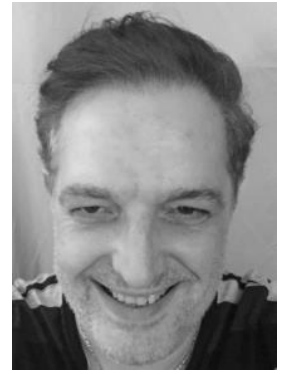

\section{Eric Céret}

est Maître de Conférences en Informatique à l'Université Grenoble Alpes. Après 20 dans l'industrie du logiciel comme ingénieur et chef de projet, il a soutenu sa thèse en 2014, sur la flexibilité des méthodes de développement pour les IHM. En 2015, il a été recruté comme maître de conférences. II focalise ses recherches sur la modélisation de l'utilisateur au service de l'adaptation et de la personnalisation des IHM. Plus particulièrement, il étudie les aspects dynamiques de ce modèle, c'est-à-dire les possibilités de représenter un utilisateur changeant et mobile. Enfin, il examine comment prendre en compte cette représentation pour offrir une IHM correspondant au mieux aux attentes de l'utilisateur.

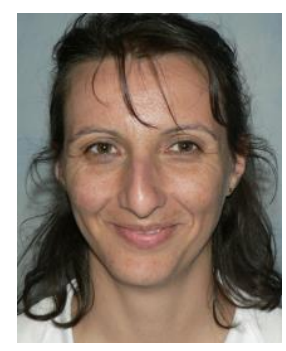

\section{Sophie Dupuy-Chessa}

est Professeure en Informatique à l'Université Grenoble Alpes depuis 2014. Elle a obtenu sa thèse à Grenoble en 2000 dans le domaine du génie logiciel, plus précisément en modélisation. Puis elle a été chargée de cours à l'Université de Genève et chargée de recherche au Xerox Research Center Europe. En 2002, elle a été recrutée comme Maître de conférences à l'Université Pierre Mendès-France où elle a développé ses recherches à la croisée des chemins entre interaction homme-machine et systèmes d'information. Ses activités actuelles portent sur la qualité des systèmes d'information innovants en se focalisant sur l'expérience utilisateur par l'adaptation des interfaces homme-machine.

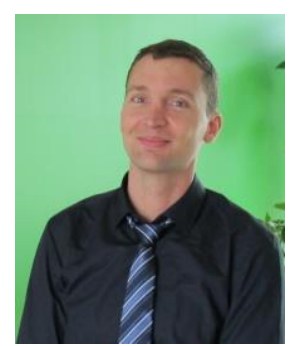

\section{Jean-Philippe Poli}

est ingénieur-chercheur au CEA List depuis 2007 et enseignant à l'école Centrale Supélec depuis 2004. II a soutenu sa thèse en 2007 sur l'indexation automatique des flux télévisuels à l'Institut National de l'Audiovisuel et l'Université Paul Cézanne. En 2007, il est recruté au CEA pour travailler sur des projets sécurité pour lesquels il développe un système expert flou nouvelle génération, appelé ExpressIF. Ses recherches portent aujourd'hui sur la représentation des connaissances vagues, l'apprentissage de modèles interprétables et l'explicabilité des décisions. Les applications de ses travaux sont nombreuses, comme par exemple la médecine personnalisée, la sécurité et la plasticité des interfaces. 